\title{
Axonal Recordings from Medial Superior Olive Neurons Obtained from the Lateral Lemniscus of the Chinchilla (Chinchilla laniger)
}

\author{
Peter Bremen and Philip X. Joris \\ Laboratory of Auditory Neurophysiology, Department of Neurosciences, University of Leuven, 3000 Leuven, Belgium
}

Interaural time differences (ITDs) are a major cue for localizing low-frequency $(<1.5 \mathrm{kHz})$ sounds. Sensitivity to this cue first occurs in the medial superior olive (MSO), which is thought to perform a coincidence analysis on its monaural inputs. Extracellular single-neuron recordings in MSO are difficult to obtain because (1) MSO action potentials are small and (2) a large field potential locked to the stimulus waveform hampers spike isolation. Consequently, only a limited number of studies report MSO data, and even in these studies data are limited in the variety of stimuli used, in the number of neurons studied, and in spike isolation. More high-quality data are needed to better understand the mechanisms underlying neuronal ITD-sensitivity.

We circumvented these difficulties by recording from the axons of MSO neurons in the lateral lemniscus (LL) of the chinchilla, a species with pronounced low-frequency sensitivity. Employing sharp glass electrodes we successfully recorded from neurons with ITD sensitivity: the location, response properties, latency, and spike shape were consistent with an MSO axonal origin. The main difficulty encountered was mechanical stability. We obtained responses to binaural beats and dichotic noise bursts to characterize the best delay versus characteristic frequency distribution, and compared the data to recordings we obtained in the inferior colliculus (IC). In contrast to most reports in other rodents, many best delays were close to zero ITD, both in MSO and IC, with a majority of the neurons recorded in the LL firing maximally within the presumed ethological ITD range.

\section{Introduction}

To localize low-frequency $(<1.5 \mathrm{kHz})$ sounds in the horizontal plane, mammals exploit minute interaural time differences (ITDs) between the sound waves at the two ears. There is general agreement that coincidence detection and internal delays are involved in the neural extraction of ITD, but the source of internal delays and the nature of the neural code are a matter of ongoing debate. Several mechanisms have been proposed from simple axonal (Jeffress, 1948) or cochlear (Schroeder, 1977; Shamma et al., 1989; Joris et al., 2006) delays to precisely timed interactions between excitatory and inhibitory inputs (Grothe, 2003) or differences in rise times of contralateral and ipsilateral synaptic events (Jercog et al., 2010). The common denominator of all these models is the site at which ITD sensitivity emerges: the brainstem medial superior olive (MSO). This nucleus is located deep within the brainstem and is characterized by the following:

Received April 9, 2013; revised Sept. 23, 2013; accepted Sept. 26, 2013.

Author contributions: P.B. and P.X.J. designed research; P.B. and P.X.J. performed research; P.B. and P.X.J. contributed unpublished reagents/analytic tools; $P . B$. analyzed data; P.B. and P.X.J. wrote the paper.

P.X.J. was supported by grants from Fonds voor Wetenschappelijk Onderzoek (G.0714.09, G.0A11.13), BOF (OT/09/50; Flanders, Belgium), and P.B. by a BOF fellowship. We thank Roel Vlayen for expert help with histological processing, Eric Verschooten for assistance with cochlear microphonic measurements, and Ellen Winckelmans for participation in the IC recordings.

The authors declare no competing financial interests.

Correspondence should be addressed to Philip X. Joris, Laboratory of Auditory Neurophysiology, Campus Gasthuisberg 0/N2, K.U. Leuven, Herestraat 49 bus 1021, B-3000 Leuven, Belgium. E-mail: Philip. Joris@med.kuleuven.be.

DOI:10.1523/JNEUROSCI.1518-13.2013

Copyright $\odot 2013$ the authors $\quad 0270-6474 / 13 / 3317506-13 \$ 15.00 / 0$
(1) very small somatic action potentials (Scott et al., 2010) and (2) a stimulus-locked field potential, the neurophonic (Guinan et al., 1972; McLaughlin et al., 2010) that hampers clean single-neuron isolation and makes the use of complex stimuli problematic. Combined, these characteristics render the MSO a difficult nucleus for extracellular recordings and have led to the anomalous situation that, of all main subthalamic auditory nuclei, the MSO is the nucleus whose function is supposedly best understood and most frequently modeled, but which is at the same time one of the physiologically most poorly characterized nuclei.

Most in vivo studies dealing with the representation of ITDs are performed in the midbrain inferior colliculus (IC), which is commonly believed to inherit its ITD sensitivity via direct projections from the MSO (Winer and Schreiner, 2005). However, the IC does not exclusively receive projections from the MSO. In addition to intracollicular connections, various nuclei of the cochlear nuclear complex $(\mathrm{CN})$, the superior olivary complex (SOC), and the lateral lemniscal nuclei (LLN) project to the IC. For a better understanding of the mechanisms of ITD sensitivity, it is therefore imperative to obtain clean single-neuron data from the first site of binaural convergence: the MSO. This was the aim of the present study.

We circumvented the problems associated with direct MSO approaches by recording with sharp glass pipettes from axons ascending in the lateral lemniscus (LL) of the chinchilla. The LL is a fiber bundle containing ascending axons from the $\mathrm{CN}$, the SOC, and the LLN (Fig. 1). Since there is little published data on ITD sensitivity in the IC of the chinchilla (Chen and Sinex, 1999) we 


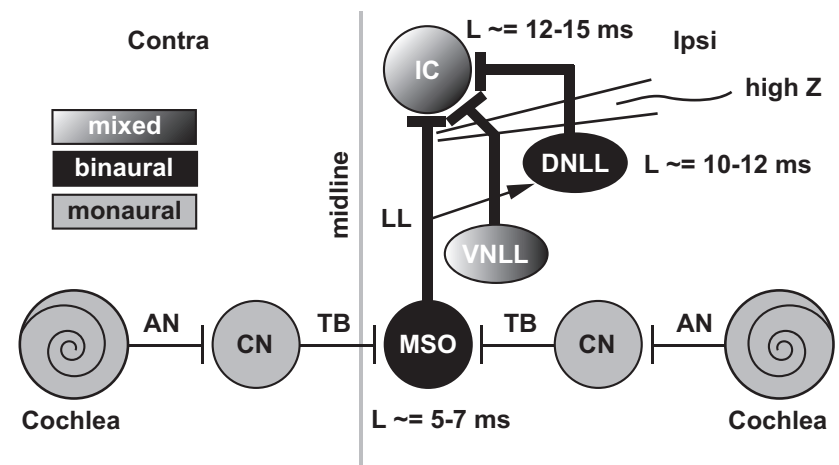

Figure 1. Schematic brainstem circuitry depicting some of the relevant nuclei for this study. Monaural and binaural structures are indicated in light gray and black, respectively. The brainstem VNLL and the midbrain IC are mixed structures with monaural as well as binaural neurons. High-impedance (high Z) glass electrodes were inserted in the LL at or above the level of the DNLL in an effort to record from MSO axons. Note that the DNLL is a binaural nucleus whose axons also run in the LL. However, recordings from MSO and DNLL axons may be differentiated by a difference in latency (L). Another binaural nucleus, the LSO, as well as monaural projections from CN and SOC are not shown in the diagram. AN, auditory nerve; TB, trapezoid body.

also recorded from this nucleus to have a reference dataset. Our distributions of best delay (BD), i.e., the ITD at which response rate is highest, are markedly different from most (McAlpine et al., 2001; Brand et al., 2002; Pecka et al., 2008, 2010) but not all (Day and Semple, 2011) studies of other rodents. Surprisingly, a large fraction of BDs in both nuclei fell within the ethological ITD range of the chinchilla.

\section{Materials and Methods}

Animal preparation. All procedures were approved by the KU Leuven Ethics Committee for Animal Experiments and were in accordance with the National Institutes of Health Guide for the Care and Use of Laboratory Animals. Single-unit recordings were obtained from a total of 54 $\left(N_{\text {LL }}=39 ; N_{\text {IC }}=15\right)$ chinchillas (Chinchilla laniger) of either sex, weighing between 300 and $500 \mathrm{~g}$. At the beginning of each experiment we intramuscularly administered a mixture of ketamine $(0.2 \mathrm{ml} / \mathrm{kg}, 100 \mathrm{mg} /$ $\mathrm{ml}$, Anesketin) and $2 \%$ xylazine $(0.15 \mathrm{ml} / \mathrm{kg}$, Rompun) to induce anesthesia and $0.08 \mathrm{ml} / \mathrm{kg}$ of atropine $(0.5 \mathrm{mg} / \mathrm{ml})$ to reduce salivation. Throughout surgical preparation and recording, the animal was kept in an areflexive state (toe-withdrawal reflex) with a 2:1 mixture of ketamine and diazepam $(5 \mathrm{mg} / \mathrm{ml}$, Valium). We used this mixture rather than a combination of ketamine and xylazine because diazepam has a larger therapeutic range than xylazine making it easier to use and because diazepam counteracts hypertonia induced by ketamine. This latter aspect is important in relation to the stability of the recordings. It should be noted that diazepam potentiates GABA-mediated inhibition, as does pentobarbital, which has been shown to influence the ITD tuning of IC neurons in the rabbit (Kuwada et al., 1989). This is likely of less importance for the LL recordings since glycine is the predominant inhibitory transmitter in the SOC nuclei of interest (Helfert et al., 1989).

Animals were placed in a double-walled sound-attenuated chamber (IAC) on a homeothermic blanket control unit (Harvard Medical Instruments) that kept body temperature at $37^{\circ} \mathrm{C}$. After removal of both pinnas and exposure of the skull a small metal rod was mounted on the frontal part of the skull with dental cement $\left(3 \mathrm{M}\right.$, clearfil $\mathrm{S}^{3}$ bond) to secure the head in a stereotaxic device. We suspended the upper body of the animal in the air in such a way that only the lower body rested on the heating pad. This suspension technique ameliorated breathing artifacts and thus increased recording stability. To equalize pressure between the middle and external ears, the bullas were vented with $30 \mathrm{~cm}$ long polyethylene tubes with an inner diameter of $0.58 \mathrm{~mm}$ (PE 50, Intramedic Clay Adams). We also routinely opened the cisterna magna to reduce the pressure exerted on the brainstem thus decreasing cardiac pulsation artifacts.
Two types of exposures were performed to approach the LL: (1) a lateral approach (17 animals) and (2) a caudal (posterior fossa) approach (22 animals). For the lateral approach the dorsal part of both bullas was opened, exposing the middle ear cavity. Although only a single bulla needed to be opened to gain access to the brainstem, a symmetrical craniotomy was performed to preserve acoustical symmetry, i.e., to avoid binaural differences generated by unequal acoustic transfer functions between sound source and inner ear. Via the window made in the right bulla, a small hole was made in the medial wall of the bulla with a scalpel blade to expose part of the cerebellum and occipital cortex. We then aspirated a small part of the cerebellum and cortex to reach the LL. This had to be done carefully to avoid hitting a dorsoventrally running plexus of blood vessels that stemmed from the transversal sinus. The caudal approach entailed opening the skull above the vermis of the cerebellum and a partial cerebellectomy to expose the LL and the ventral part of the IC. This exposure could be readily performed making use of the fourth ventricle as a landmark.

The IC was also approached in one of two ways: (1) a dorsal approach (4 animals) for which part of the right occipital cortex needed to be aspirated after making a small hole aligned to the sagittal and lambdoid sutures and the medial aspect of the bulla and (2) a caudal approach (11 animals) similar to the caudal one used to reach the LL. To reach the dorsolateral part of the IC, the region in which ITD-sensitive units are located, we had to enlarge the trepanation in the caudal direction so that we could insert the electrode under a shallow angle. Note that the bullas remained intact in both procedures.

After advancing the electrode a couple of hundred micrometers into the brain tissue we filled the hole left by the aspiration with $1.5 \%$ agar dissolved in distilled water to prevent desiccation and to suppress pulsation.

Stimulus generation. Stimuli were generated digitally with TDT System II (Tucker-Davis Technologies) using custom-written software. Digitally generated sounds were converted to analog (Power DAC) signals, attenuated (PA4, TDT System3) and delivered via dynamic speakers (Supertweeter; Radio Shack). The speakers were coupled to ear bars, which were inserted into the bony auditory meatus with a custom-made coupler and directed toward the tympanic membrane. The coupler was sealed to the bony meatus with Audalin ear impression compound (Microsonic). The system was calibrated in amplitude and phase with a $1 / 2$-inch condenser microphone (Bruël \& Kjaer).

Estimation of acoustical cross talk for the lateral approach. The lateral approach entails opening of both bullas. Opening both bullas rather than only the bulla on the side of recording has the advantage of maintaining symmetry in the acoustic transfer functions (verified by acoustic calibrations; Rosowski et al., 2006), but has the disadvantage that it may reduce interaural separation. To assess possible acoustical cross talk, we measured cochlear microphonic (CM) potentials of both ears simultaneously with silver ball electrodes placed at the round window of one chinchilla at 10 frequencies spanning a range from 100 to $2133 \mathrm{~Hz}$. Recordings were first made with both bullas closed (using ear impression compound to close the small openings necessary to place the round window electrodes), then with both bullas open. At most frequencies, the interaural attenuation was surprisingly large $(>40 \mathrm{~dB})$ and not noticeably affected by opening of the bullas. As expected, the interaural attenuation was smallest at the lowest frequencies ( 100 and $233 \mathrm{~Hz}$, where it could be as low as $30 \mathrm{~dB})$.

In addition to the CM measurements we also tested monaural, contralaterally driven fibers that we sporadically encountered and that could, for example, stem from the ventral nucleus of the lateral lemniscus (VNLL) or cochlear nucleus (CN). In none of these units did we observe an increase in firing rate above spontaneous rate after ipsilateral stimulation (broadband noise or tones in the range of 110-20,000 Hz tested up to $70 \mathrm{~dB}$ sound pressure level; SPL). We therefore conclude that the symmetric opening of both bullas only marginally affects cross talk. Accordingly, we pooled the data obtained from the lateral and caudal approaches.

Electrophysiology and data collection. We recorded from single units in the LL using micropipettes filled with $3 \mathrm{M} \mathrm{NaCl}$. We used electrodes with low impedances $(Z \simeq 1-5 \mathrm{M} \Omega)$ for scouting and sharp, high impedance 
electrodes $(Z \simeq 20-60 \mathrm{M} \Omega$ ) to record from axons. Recordings from single units in the IC were either done with low impedance $(Z \simeq 1-5$ $\mathrm{M} \Omega)$ glass micropipettes or home-made Indium electrodes $(Z \simeq 7-10$ $M \Omega$ ). In the initial experiments we used a Narishige 6-axis micromanipulator mounted on a stereotaxic device to position the electrodes. However, to increase the stability of the recordings in later experiments we fixed a Delrin chamber with dental acrylic directly to the skull of the animal on which the hydraulic microdrive (Trent Wells; ) could be mounted. This technique is similar to the one routinely used in our lab to record from auditory nerve fibers.

Extracellular voltage signals were amplified (for low-impedance electrodes: SRS560, Stanford Research Systems, 1000×, bandpass filtered $0.3-3 \mathrm{kHz}$, roll-off $6 \mathrm{~dB}$ and for high-impedance electrodes: Molecular Devices Axoclamp 2B Intracellular amplifier and an additional PAR amplifier with variable gain setting, bandpass filtered $0.3-3 \mathrm{kHz}$, roll-off 6 $\mathrm{dB}$ ), windowed (early experiments: custom-built peak picker; later experiments: window-discriminator from Bak Electronics) and time stamped with a resolution of $1 \mu$ s before being digitized (TDT system II) and stored. In 15 LL experiments and two IC experiments we also sampled and stored the spike waveforms, sound waveforms, and the BAK trigger pulses using an RX8 (sampling frequency $50 \mathrm{kHz}$ or 100 $\mathrm{kHz}, \mathrm{RX} 8$, TDT system 3) and custom-written software (MATLAB; The MathWorks).

While advancing the electrode through the brain tissue either pure tones $(110-20,000 \mathrm{~Hz}, 250 \mathrm{~ms}$ with a silent interval of $50 \mathrm{~ms}$ at $70 \mathrm{~dB}$ SPL) or binaural beats ( $f_{\text {beat }}=2 \mathrm{~Hz}, 5 \mathrm{~s}$ with a silent interval of $1 \mathrm{~s}$ at 70 $\mathrm{dB}$ SPL) were presented binaurally to search for auditory units. When a unit was isolated, we recorded a binaural frequency tuning curve (FTC) at zero ITD with a threshold tracking algorithm (resolution: 0.1 octave steps) to determine its characteristic frequency $(\mathrm{CF})$. Next, we presented binaural beats $\left(\mathrm{BB}\right.$; level $=70 \mathrm{~dB} \mathrm{SPL}, f_{\text {beat }}=2 \mathrm{~Hz}$, duration $=5 \mathrm{~s}, N_{\text {rep }}$ $=3$ ) over a range of carrier frequencies $\left(f_{\text {car }}\right)$ based on the FTC. Subsequently, we collected noise delay functions (NDFs) to correlated $(\rho=1)$ and anti-correlated $(\rho=-1)$ broadband noise bursts $(50-8000 \mathrm{~Hz}, 6 \mathrm{~ms}$ $\cos ^{2}$ rise/fall time) of a duration of $1 \mathrm{~s}$ or $5 \mathrm{~s}$ at a level of $70 \mathrm{~dB}$ SPL varying in ITD between -5 and $5 \mathrm{~ms}$ in steps of $0.5 \mathrm{~ms}$ with three repetitions per condition. Presentation of ITDs was randomized. If stability allowed we collected NDFs with a higher resolution.

The advantage of using binaural beats as a search stimulus is that it immediately reveals interaural temporal sensitivity to the fine-structure of the sound waveform, in the form of "beating" of the spike rate to the ongoing and changing interaural phase difference (IPD) between the sounds to the two ears (Kuwada et al., 1979). A limitation of the binaural beat stimulus is that it does not reveal interaural temporal sensitivity to stimulus envelope, but such sensitivity should be revealed by properties of the NDFs (Joris, 2003). Another limitation is that sensitivity to binaural beats is not restricted to MSO (Yin and Chan, 1990; Spitzer and Semple, 1995; Day and Semple, 2011; van der Heijden et al., 2013) but has also been reported in lateral superior olive (LSO) neurons (Joris and Yin, 1995; Spitzer and Semple, 1995; Tollin and Yin, 2005). As mentioned below (see below, Binaural beat analysis), detailed analysis of binaural beat responses over a range of frequencies allows tentative but not conclusive identification of MSO versus LSO responses. Other issues can be raised such as the sensitivity to low-frequency ITDs in periolivary nuclei of the SOC (Goldberg and Brown, 1969; Spitzer and Semple, 1995; Day and Semple, 2011), in VNLL (Batra and Fitzpatrick, 2002), and in nonprimary coincidence detectors (Goldberg and Brown, 1969; Spitzer and Semple, 1995; Batra et al., 1997; Coffey et al., 2006). Consideration of the known response properties of MSO and non-MSO neurons with ITD sensitivity argues that the bulk of our data is consistent with an MSO origin rather than with a non-MSO origin: we thus label the ITD sensitivity reported here as "originating in MSO" but this is tentative (see Discussion).

Histological reconstruction of the recording site. To reconstruct the recording site the electrode track was marked either electrolytically (Indium electrodes, $10 \mathrm{~s}$ of $10 \mu \mathrm{A}$ negative pulsating current at the last site of clear auditory activity, Midgard Precision Current Source; Stoelting) or with $1,1^{\prime}$-dioctadecyl-3,3,3'3' -tetramethylindocarbocyanine perchlorate, (DiI; Invitrogen) applied to the glass electrodes before electrode

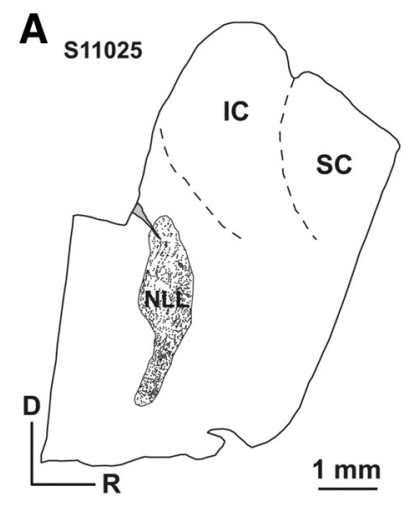

B

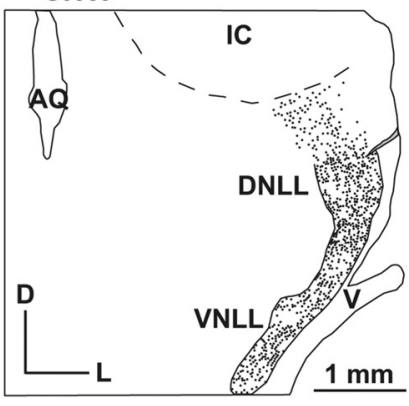

Figure 2. Histological reconstruction of recording sites of two animals. Overlays of tracings of fluorescent images and cresyl violet stained sections. $\boldsymbol{A}$, Caudal approach, parasagittal view. $\boldsymbol{B}$, Lateral approach, coronal view. AQ, aqueductus; $D$ dorsal; L, lateral; NLL, nuclei of the lateral lemniscus; $R$, rostral; SC, superior colliculus; $V$, trigeminal nerve. Electrode tracks are marked in light gray with black outlines. The VNLL and DNLL were first outlined; within those outlines locations of nucleated cell bodies are indicated with a dot (see main text).

placement. DiI is a hydrophobic cyanine dye that can be used to mark cell membranes (DiCarlo et al., 1996).

After the experiment, animals were killed with an overdose of sodium pentobarbital and perfused transcardially with $10 \%$ formalin. After cryoprotection in a $30 \%$ sucrose solution for at least $1 \mathrm{~d}$, the brainstem was frozen using dry ice and sectioned $(50 \mu \mathrm{m})$ either sagittally (caudal and dorsal approaches) or transversely (lateral approach) using a sliding microtome (HM430; Microm). All sections were first viewed under a fluorescent microscope (Olympus BX-61 with a focus control unit BX-UCB and a fluorescent light source X-Cite 120Q) filtered at $565 \mathrm{~nm}$ to visualize the DiI signal. After tracing and photographing the fluorescent signal and the outline of the brainstem (Neurolucida; MBF Bioscience) standard Nissl staining was performed to visualize brainstem nuclei. Photographs of the stained sections were taken and digital overlays with the DiI photographs were made and traced (Adobe Photoshop).

Analysis. Unless otherwise stated, all fits used the least-squares criterion.

Spike shape. It is a general observation that monopolar spikes are associated with axonal recordings and bipolar spikes with somatic recordings. This is in line with our own observation in recordings from auditory nuclei (e.g., $\mathrm{CN}$ or IC) versus recordings from axon bundles (e.g., auditory nerve or trapezoid body). We recorded the spike waveforms in $15 \mathrm{LL}$ experiments. We first determined the mean spike waveform over all stimuli and repetitions per dataset, i.e., per BB, NDF, etc., by averaging the spikes over a window of $8 \mathrm{~ms}$ centered on the occurrence of the trigger pulse of the spike discriminator. To classify the spike waveforms as being either monopolar, i.e., axonal, or bipolar, i.e., somatic, we calculated the polarity index (PI) as follows:

$$
P I=\left(V_{\max }-V_{\min }\right) / V_{\max },
$$

with $V_{\max }$ being the maximal voltage and $V_{\min }$ the minimal voltage of the mean spike waveform. After visual inspection of the PI distribution we choose values $\leq 1.6$ to indicate a monopolar spike waveform (see Fig. 5).

Latency analysis. Because our tracks were dorsal to or transversed the dorsal nucleus LL (DNLL; Fig. 2), axonal recordings may also stem from ITD-sensitive DNLL neurons projecting to the IC. Therefore, we subjected the two classes of spike shapes, i.e., monopolar and bipolar, to a latency analysis. The rationale for this analysis is that the DNLL is at least one synapse "higher" in the ascending auditory pathway than MSO neurons (Fig. 1). Accordingly, if spikes stem from SOC axons, monopolar spikes should exhibit shorter latencies than bipolar spikes. Moreover, if the recordings are indeed of SOC origin and are not mixed with axons from the DNLL, we expect the latency distribution of monopolar spikes to be unimodal.

We compared response latencies in two ways by tallying the first spikes after stimulus onset, and by constructing poststimulus time histograms (PSTHs). For each neuron, both methods were applied across all stimu- 
lus conditions and repetitions, resulting in a single latency profile per neuron for each method. Normalization was performed so that the area of each profile was equal to one per neuron. After summing the individual distributions separately for monopolar and bipolar datasets, we normalized the summed distribution again so that the area under the curve equaled one: these are the profiles shown in Figure $5 D$ and $E$. No effort was made to remove "spontaneous" spikes from contributing to the first-spike latency data. As will be discussed in Results (Fig. $5 D, E$ ), the contribution of early "spontaneous" spikes was small.

FTCs. The highest CFs we encountered were 12.3 and $4.3 \mathrm{kHz}$ in the LL and IC, respectively. We focused on ITD sensitivity to binaural beats at and near CF: such ITD sensitivity was only observed in units with a CF below $1500 \mathrm{~Hz}$. We therefore restrict analysis in this paper to units with CF below that limit. We did not study ITD sensitivity in the lowfrequency "tail" of neurons with higher CF.

We only included FTCs that had a minimal threshold of at most $65 \mathrm{~dB}$ SPL. This criterion led to the exclusion of three and four neurons in the LL and IC, respectively. The next criteria for inclusion in our database were based on a rounded exponential filter (roex-filter; Patterson, 1976) fit to the FTCs. The symmetrical roex-filter is an estimate of the shape of the auditory filter, and is parameterized as follows:

$$
|H(f)|^{2}=(1+p g) e^{-p g},
$$

where $g$ is the normalized deviation of the frequency from the characteristic frequency CF, $p$ is the slope parameter, and $|H(f)|^{2}$ represents the squared magnitude of the filter. Parameter $p$ can be used to calculate the equivalent rectangular bandwidth (ERB) as follows:

$$
E R B=4 \mathrm{CF} / p .
$$

In addition to the threshold criterion, an FTC had to satisfy the two following criteria: its ERB had to exceed $40 \mathrm{~Hz}$ and the coefficient of determination $\left(R^{2}\right)$ of the roex-fit had to be larger than 0.8 . These criteria allowed automated rejection of oddly shaped tuning curves.

We smoothed the FTCs depicted in Figure 6 with a moving average of 5 points for display purposes.

Binaural beat analysis. We characterized the phase-locking features of a unit with a standard vector-averaging approach (Goldberg and Brown, 1969). Responses were binned (period histogram) at the beat period for all $f_{\text {car }}$ and the mean phase was calculated according to the following:

$$
y=\sum_{j=1}^{n} \sin \left(2 \pi f t_{j}\right)
$$

and

$$
x=\sum_{j=1}^{n} \cos \left(2 \pi f t_{j}\right),
$$

with $f$ being the beat frequency in $\mathrm{Hz}, t$ the time of occurrence of the $j$ th spike, and $n$ the number of spikes. Individual spikes are considered to be vectors distributed along the unit circle and the direction, i.e., the phase $\varphi$, of the mean of this distribution is given by the following:

$$
\varphi=\arctan \left(\frac{y}{x}\right)+k \pi
$$

where $k$ equals zero or one depending on the signs of $x$ and $y$.

The vector strength (VS), i.e., the amplitude of the vector, was then calculated as follows:

$$
\mathrm{VS}=\frac{\sqrt{x^{2}+y^{2}}}{n} .
$$

A VS of 1 indicates perfect phase locking; no phase preference gives a VS near 0 . We tested the significance of phase locking with the Rayleigh test of uniformity (Mardia, 1972):

$$
p=\mathrm{e}^{\left(-\alpha(V S)^{2}\right)} .
$$

We considered $p \leq 0.001$ to indicate significant phase locking. Only data that confirmed to this criterion were included in the analysis. Subsequently, we fit a regression line through the $\varphi$ versus $f_{\text {car }}$ data weighing each data point with the product of its VS and spike rate (Kuwada et al., 1987). The slope of the regression line gives the characteristic delay (CD) and the $y$-intercept yields the characteristic phase (CP) of the unit. The $\mathrm{CP}$ indicates whether tone delay curves would coincide at a peak $(\mathrm{CP} \sim$ $0)$, at a trough $(\mathrm{CP} \sim 0.5)$, or somewhere between a peak and a trough (CPs other than $0,0.5$, and 1 ) and can thus be used to approximately classify units as peak type or trough type (Batra et al., 1997). As far as known, MSO neurons are mostly peak type and LSO neurons mostly trough type, but the $\mathrm{CP}$ is not absolutely dichotomous as a criterion (Yin and Chan, 1990; Joris and Yin, 1995; Tollin and Yin, 2005; Day and Semple, 2011).

We tested for linearity of the $\varphi$ versus $f_{\text {car }}$ relation by using a statistical approach based on the sum of squares of the deviation of the points from the regression line (Yin and Kuwada, 1983, their Appendix). Only units that satisfied the linearity criterion were included in the analysis. Additionally, we excluded weakly driven units, i.e., whose maximal spike rate was $<10$ spikes/s.

Furthermore, we calculated so-called composite curves to estimate a neuron's BD to wideband stimuli. First, we converted the BB responses to tone delay functions (TDFs) for all $f_{\text {car }}$ tested by multiplying the phase of the period histogram with the period of the corresponding $f_{\text {car }}$. The composite curve is obtained by linearly summing the TDFs: it is a linear estimate of the response to a broadband (covering the tested $f_{\text {car }}$ s) stimulus, e.g., of the NDF to broadband correlated noise.

Noise delay functions. We classified a unit as being ITD sensitive to broadband noise if it met three different requirements. First, we calculated the modulation depth $\left(M D_{N D F}\right)$ for each NDF as follows:

$$
M D_{N D F}=\left(R_{\max }-R_{\min }\right) / R_{\max },
$$

with $R_{\max }$ and $R_{\min }$ being the maximal response and minimal response of the NDF, respectively (Joris, 2003; Smith and Delgutte, 2007). Next, each NDF was fit with two different functions:

$$
\text { Rate }=A^{\star} \sin \left(I T D^{\star} 2 \pi^{\star} f+\varphi\right)+S,
$$

where $A(\mathrm{~Hz})$ is the amplitude, $f(\mathrm{~Hz})$ is the frequency, $\varphi(\mathrm{rad})$ is the phase, and $S(\mathrm{~Hz})$ the offset. The second function was a Gaussian, which had the form

$$
\text { Rate }=A e^{\frac{(I T D-B D)^{2}}{H R^{2}}}+B,
$$

where $A(\mathrm{~Hz})$ represents the amplitude, $B D(\mathrm{~ms})$ the best delay, $H W(\mathrm{~ms})$ the half-width, and $B$ the offset $(\mathrm{Hz})$. A unit was deemed to be ITD sensitive if its $M D$ was at least 0.7 , its maximal firing rate exceeded 10 spikes/s, and if at least one of the two fits had a coefficient of determination $\left(R^{2}\right)$ above 0.8 . These criteria ensured the exclusion of neurons with poor ITD sensitivity.

Measurement of $B D$. For both the responses to binaural beats and noise, we determined the delay yielding the maximal response, i.e., the $\mathrm{BD}$. Because it is a key value, we tried to incorporate as much data as possible in the BD measurements. For units for which NDFs to both correlated and anti-correlated noise were available, we calculated the difference between them. The resulting curve is called the "difcor" (Joris, 2003). The difcor is less noisy than NDFs and provides a simple response linearization. From the fast Fourier transform of the difcor the so-called dominant frequency (DF) can be derived (Joris et al., 2005).

We also obtained a difcor from the responses to tones. Besides the composite curve obtained by linear summation of the TDFs, we constructed a composite curve to responses phase shifted by $\pi$ before summing, and the two composite curves were then subtracted just as for the NDFs (Joris et al., 2005). For all ITD curves (TDFs, NDFs, and composite curves) positive ITD values indicate a lead in the contralateral ear (i.e., re recording site): a condition which in the free field normally corresponds to contralaterally positioned sound sources.

An important aim of our recordings was to study the distribution of $\mathrm{BD}$ as a function of CF. However, CF was not available for all neurons: in 
those cases we used DF, calculated from the difcor, as a substitute value. It has been shown earlier that the DF derived from NDFs is well correlated to CF up to several kilohertz, both in monaural neurons (auditory nerve: Louage et al., 2004, trapezoid body: Louage et al., 2005) and binaural neurons (Joris et al., 2005). Another frequently used measure is best frequency (BF; i.e., the frequency for which response to tones is maximal), but the correlation of that measure to CF is poor: bushy cells (Joris et al., 1994a,b) and binaural neurons (Joris et al., 2005) commonly show much higher firing rates at low frequencies, in the tail of their tuning curves, than at CF. For the population of neurons for which both measures were available, $R^{2}$ between CF and DF, based on NDFs, was 0.92 $(p \ll 0.01, N=24)$. BF, however, was poorly correlated to $\mathrm{CF}\left(R^{2}=\right.$ $0.66, p=0.01, N=14)$ and was not used here.

\section{Results}

We present data from a total of 177 neurons recorded in the LL and 68 neurons from the IC. A total of 141 FTCs were recorded in the LL (55 lateral approach, 86 caudal approach) and 60 in the IC (47 caudal approach, 13 dorsal approach). Of those a subset of 85 LL and 33 IC neurons had CFs $<1500 \mathrm{~Hz}$ and were binaurally sensitive. We obtained responses to BB stimuli from $32 \mathrm{LL}$ (10 lateral approach, 22 caudal approach; 9 CF, 23 DF) and from 7 IC (6 caudal approach, 1 dorsal approach; $7 \mathrm{CF}, 0 \mathrm{DF}$ ) individual neurons as well as $28 \mathrm{LL}$ ( 10 lateral approach, 18 caudal approach; $13 \mathrm{CF}, 15 \mathrm{DF}$ ) and 22 IC (19 caudal approach, 3 dorsal approach; $16 \mathrm{CF}, 6 \mathrm{DF})$ neurons probed with binaurally delayed noise bursts (NDFs). Three of the 177 LL neurons appeared to be monaural when tested with ipsilateral, contralateral, and binaural tone pips at CF. Two of these neurons ( 1 chopper type and 1 onset type) were contralaterally driven and the remaining chopper-type neuron was ipsilaterally driven. The surprisingly small number of monaurally driven neurons is most likely due to a recording selection bias (e.g., in fiber diameter and/or location) combined with stability issues that prevented us from fully characterizing some encountered neurons, i.e., we could not hold all fibers long enough to measure more than an FTC (these recordings are not further considered here).

\section{Histological reconstruction of the recording site}

Figure 2 depicts tracings of a sagittal section (Fig. 2A) through part of the brainstem and midbrain and of a transverse section (Fig. 2B) of two animals, in which the electrodes were aimed at the LL. Borders of the nuclei of the LL such as the DNLL and the VNLL are marked with solid black lines. The borders of the IC and the superior colliculus are indicated with dashed black lines. Locations of neurons that showed Nissl bodies in the cresyl violet stained slices, and which were located within the nuclear boundaries (solid black lines) are depicted as black circles. The electrode tracks are visualized as light gray patches with black edges, which indicate the distribution of DiI seen under fluorescence microscopy. Typically, in both the caudal (Fig. 2A) and the lateral (Fig. $2 B$ ) approaches, electrodes penetrated the dorsal portion of the DNLL or the lemniscal axon bundle that exits the DNLL dorsally and projects to the IC, so that axonal recordings could potentially stem from DNLL output axons projecting to the IC instead of SOC axons. However, we will argue below that these two populations can be distinguished by a difference in first-spike latency. Before presenting the results of a latency analysis we first describe spike waveforms.

\section{Spike waveforms}

We routinely observed the spike waveforms on an oscilloscope, but in 15 experiments aimed at the LL we recorded not only spike times but also spike waveforms. Figure $3, A$ and $B$, shows the spike
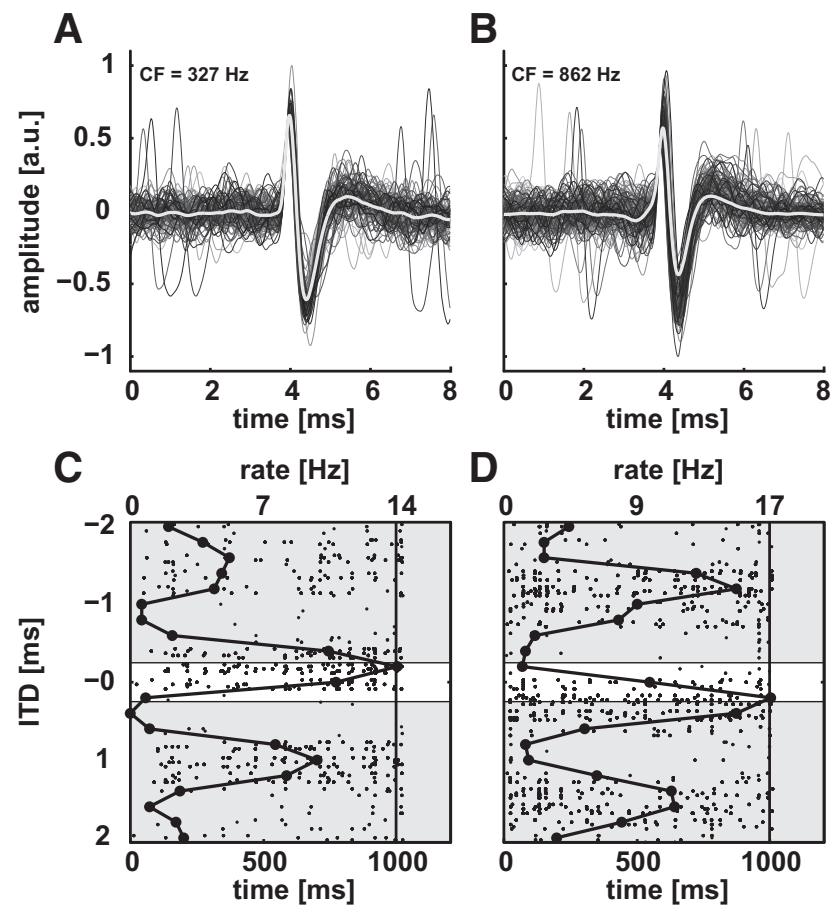

Figure 3. Bipolar spike waveforms $(\boldsymbol{A}, \boldsymbol{B})$ and dot raster plots $(\boldsymbol{C}, \boldsymbol{D})$ of two neurons recorded in the LL with low-impedance pipettes. The neurons in $\boldsymbol{A}$ and $\boldsymbol{B}$ had CFs of 327 and $862 \mathrm{~Hz}$, respectively. $\boldsymbol{A}, \boldsymbol{B}$, Individual spikes are shown in shades of gray and the mean spike shape waveform is shown as a white trace. $\boldsymbol{C}, \boldsymbol{D}$, Raster plots and noise delay functions (black line with markers, top abscissa) to correlated noise bursts for the units shown in $\boldsymbol{A}$ and $\boldsymbol{B}$. The white patches indicate the approximate ethologically experienced ITD range of the chinchilla. The solid vertical lines mark the end of the steady state of the stimulus. Both neurons were clearly sensitive to change in ITD. Note that the neuron in $\boldsymbol{A}$ and $\boldsymbol{C}$ had a negative BD within the ethological ITD range. a.u., arbitrary unit.

waveforms of neurons with CFs of $327 \mathrm{~Hz}(A)$ and $862 \mathrm{~Hz}(B)$ recorded with low-impedance electrodes and stimulated with interaurally delayed, binaural noise bursts (duration $=1000 \mathrm{~ms} ; 5$ $\mathrm{ms} \cos ^{2}$-onset/-offset ramp; $\mathrm{BW}=50-8000 \mathrm{~Hz}$; level $=70 \mathrm{~dB}$ SPL; $\left.\rho=1 ; N_{\text {rep }}=3\right)$. We extracted the waveforms $4 \mathrm{~ms}$ prior and $4 \mathrm{~ms}$ after spike occurrence (Fig. $3 A, B$, shades of gray) as determined by the BAK window discriminator and averaged the extracted shapes (thick white line). The triggered spikes shown stem from a single neuron. There are no spikes at intervals smaller than a few milliseconds. Note that both cases exhibit a large initial positive deflection followed by an equally large negative deflection (normalized amplitude given in arbitrary units, a.u.). This bipolar shape is typically associated with recordings of somatic origin, i.e., here from DNLL neurons. The corresponding dot raster plots are shown in Figure $3 C$ and $D$. In these plots each dot represents the occurrence of one spike and the stimulus parameter varied, i.e., ITD, is plotted on the ordinate with time shown on the bottom abscissa. The vertical solid line indicates the offset of the stationary part of the stimulus, i.e., up to the offset ramp. The thick line with circular symbols shows the mean spike rate in $\mathrm{Hz}$ (top abscissa), i.e., the neurons' NDF. The white patch centered at zero ITD indicates the approximate ethological range of ITDs of the chinchilla (von Bismark and Pfeiffer, 1967; Koka et al., 2011; Lupo et al., 2011). Note that the BD of both neurons falls within this range. For the response in Figure 3, $A$ and $C$, the $\mathrm{BD}$ is at negative delay whereas the other neuron's (Fig. $3 B, D$ ) $\mathrm{BD}$ is positive. As we will show later (Fig. 11) most of the recorded neurons had positive BDs, i.e., would respond best to sounds in the contralateral hemifield. 


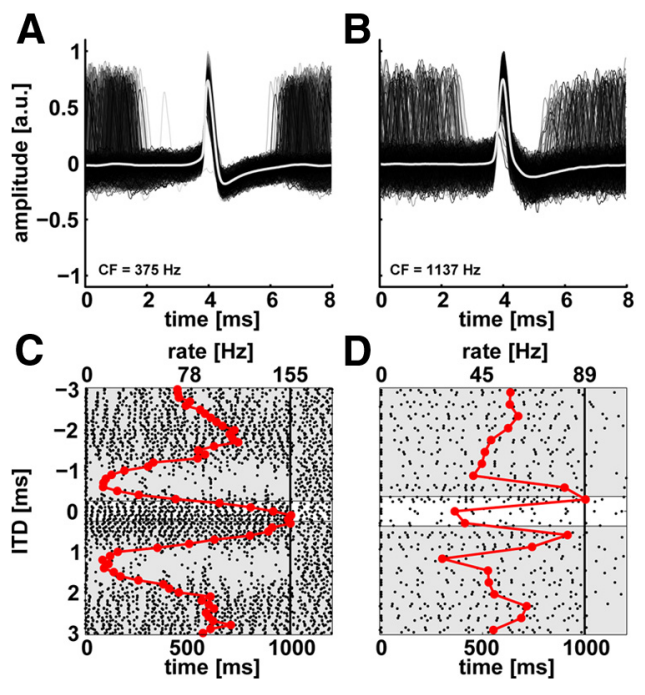

Figure 4. Monopolar spike waveforms $(\boldsymbol{A}, \boldsymbol{B})$ and dot raster plots $(\boldsymbol{C}, \boldsymbol{D})$ of two $L L$ neurons recorded with high-impedance pipettes. The neurons in $\boldsymbol{A}$ and $\boldsymbol{B}$ had CFs of 375 and $1137 \mathrm{~Hz}$, respectively. Format as in Figure 3 except that the spike rate is shown as a red line with markers. The neuron shown in $\boldsymbol{D}$ was stimulated with anti-correlated noise. Compared with the neurons in Figure 3 the firing rate is higher. a.u., arbitrary unit.

Figure 4 depicts the spike waveforms $(A$ and $B)$ and dot raster plots $(C$ and $D)$ of responses of two neurons recorded with highimpedance electrodes (left column CF $=375 \mathrm{~Hz}$ CF; right column $\mathrm{CF}=1137 \mathrm{~Hz}$ ) in response to ITDs of broadband noise. Individual spikes are shown in shades of gray and the averaged spike shape is shown as a thick white line. The recordings show a large signal-to-noise ratio and spike intervals do not violate the refractory period, again indicating that these are clean singleneuron recordings. In contrast to the spikes shown in Figure 3, $A$ and $B$, these waveforms exhibit a large positive deflection followed by a small negative deflection, i.e., they are monopolar.

We classified our data in monopolar and bipolar spikes by calculating the PI (see Materials and Methods), for all averaged spike waveforms. The resulting PI values are shown in Figure $5 \mathrm{~A}$ as a function of the difference between the time of maximal and minimal amplitude. Because there is a break at 1.6, we classified waveforms with PI equal to or smaller than 1.6 to indicate a monopolar spike (dark gray markers) and larger values to indicate bipolar spikes (white markers). The results of this classification are shown in Figure $5 B$ and $C$. These subplots show the averaged spike waveforms of all spikes classified as monopolar (Fig. 5B) and bipolar (Fig. 5C). Note that we sampled far less bipolar than monopolar waveforms (3 neurons vs 29 neurons) since we aimed at collecting data from axons with highimpedance electrodes. Impedance dropped in the course of a penetration thus biasing the recording toward a somatic spike origin, so we were occasionally able to record from axons and somata within one penetration. The neurons classified as bipolar stem from such penetrations.

\section{Latency analysis}

We extracted the first-spike latency of all monopolar and bipolar units to all stimuli and repetitions and calculated the summed probability distributions (see Materials and Methods). The resulting histograms are shown in Figure 5D. The histogram for all monopolar units is shown in dark gray, the one for bipolar units is depicted in white. It is apparent that the histogram for the bipolar, i.e., presumed DNLL recordings, peaks between 10 and
$15 \mathrm{~ms}$. The first-spike latency distribution of the monopolar spikes, however, clearly peaks earlier, between 6 and $8 \mathrm{~ms}$. As is visible in Figure 4, $C$ and $D$, "spontaneous" spikes were often present, particularly in monopolar units, and generate the low plateau of short first-spike latencies in Figure 5D. Because their contribution is small and does not hamper interpretation of the difference between monopolar and bipolar distributions, no effort was made to remove these "spontaneous" spikes from the first-spike latency calculation. A simpler analysis is shown in Figure $5 E$, which shows cumulative PSTHs for the monopolar and bipolar units. Here again, there is a clear difference in rise time and peak response for bipolar and monopolar units. Note that the absence of a second peak in both distributions of monopolar units (Fig. $5 D, E$ ) at latencies similar to the bipolar distribution also suggests that the majority of the data in that set are not of DNLL origin. There are, to our knowledge, no published latency values for chinchilla MSO or DNLL responses. However, considering the slow stimulus rise time and low CFs, the two ranges of latencies obtained here are in the right ballpark to be consistent with an SOC and DNLL origin (cat: Brugge et al., 1970; Guinan et al., 1972). The latencies are thus consistent with the interpretation that the monopolar responses largely reflect activity of axons of SOC neurons and the bipolar responses from DNLL neurons. Note, however, that the latency analysis does not allow for the disambiguation between an MSO or LSO origin.

Together the spike waveform, binaural sensitivity, and latency distributions strongly suggest that with high-impedance electrodes we indeed recorded from SOC axons. Henceforth, "LL neurons" refers to neurons generating monopolar spike waveforms (as documented here and monitored throughout all experiments by online display of spike waveforms on an oscilloscope and by impedance tests). Note that all units reported as stemming from the LL had latencies $<10 \mathrm{~ms}$. We now turn to the characterization of units recorded from the LL and the IC without further considering the three bipolar neurons of presumed DNLL origin.

\section{FTCs}

Even though CF figures prominently in discussions of binaural coding (McAlpine et al., 2001), few studies in MSO obtained threshold tuning curves and traditional CF measures, due to the recording problems in MSO mentioned earlier (see above, Introduction). Such measures were one of the aims in the present study. To assess the quality and validity of our CF measure we present an analysis of the FTCs. Figure 6 shows a manually chosen selection of tuning curves obtained in the LL $(A)$ and the IC $(B)$. These representative curves spanning the low-frequency range have been slightly smoothed by applying a running average. Markers indicate thresholds at CF for all ITD-sensitive neurons recorded, not only for those for which FTCs are shown. We also recorded from 41 and 13 neurons with $\mathrm{CF}>1500 \mathrm{~Hz}$ in the LL (12.3 kHz, highest CF encountered) and IC ( $4.3 \mathrm{kHz}$, highest CF encountered), respectively. These neurons were not sensitive to IPD in binaural beats at frequencies around CF and are not further considered here. Possibly these neurons had ITD sensitivity in their low-frequency tail or to envelopes (e.g., using broadband noise or amplitude-modulated sounds), but this was not systematically explored, mostly for reasons of time.

The most striking observation here is the abundance of well tuned and sensitive neurons with CFs as low as a few hundred hertz. The histograms of Figure 6, $C$ and $D$, provide an overview of the normalized frequency and threshold distributions of the LL (red) and IC (blue). From Figure 6 it becomes clear that we en- 
A

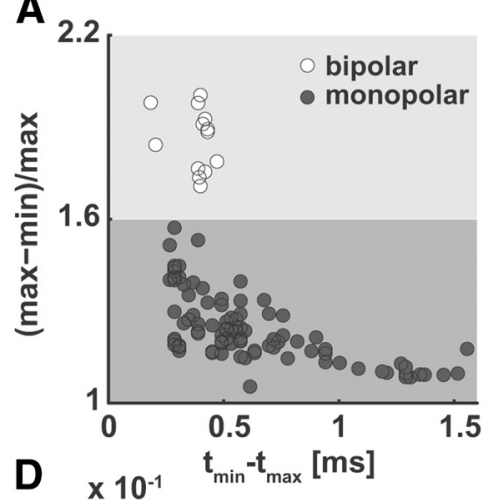

$\begin{array}{ll}\times 10^{-1} & \mathbf{t}_{\text {min }}{ }^{-t_{\text {max }}}[\mathrm{ms}] \\ 2.5 & \mathrm{~N}_{\mathrm{bi}}=14 / 3\end{array}$

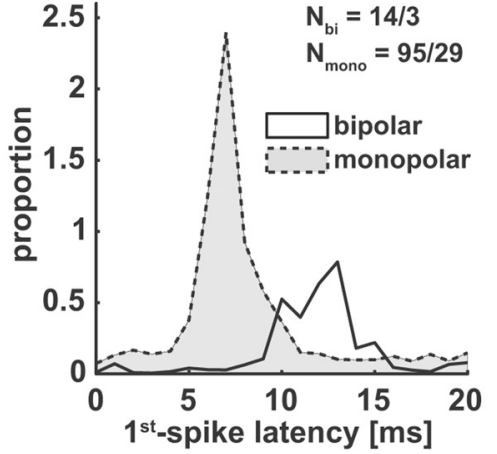

B
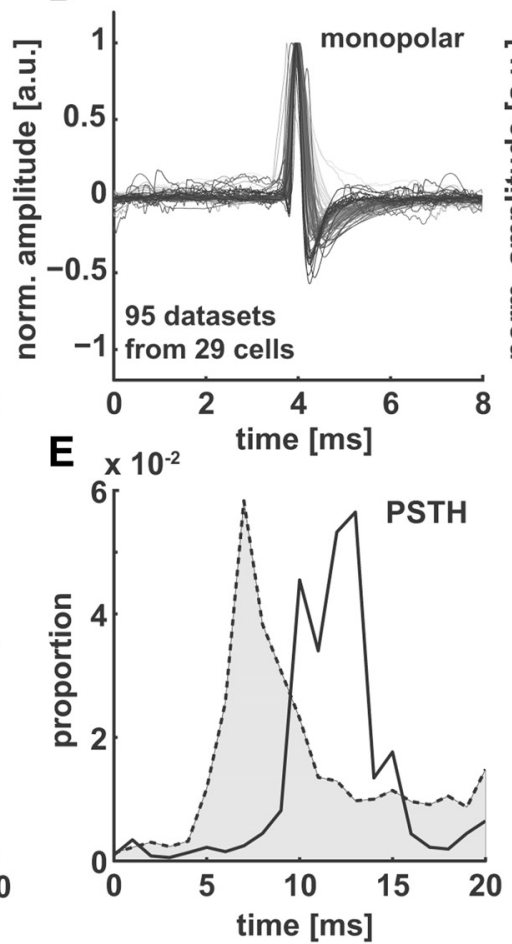

C

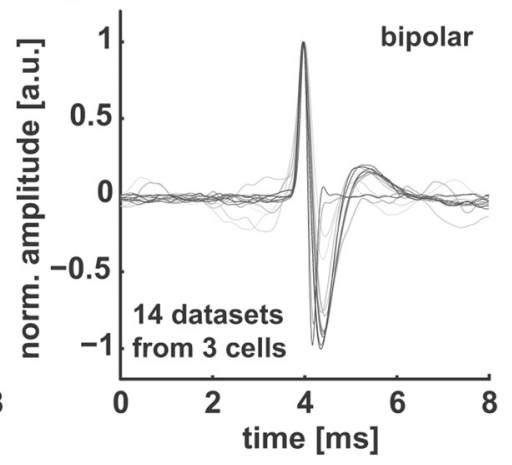

Figure 5. A, Polarity index (PI) as a function of the difference between time-to-minimum and time-to-maximum. Dark gray and white circles indicate data from recordings exhibiting monopolar and bipolar spike waveforms, respectively. A PI of 1.6 was the criterion for separation between monopolar and bipolar waveforms. We used this value to classify the data and the results are shown in $\boldsymbol{B}$ and $\boldsymbol{C}$. These subplots show the averaged spike waveforms for 95 stimulus datasets of 29 neurons classified as having monopolar spike waveforms $(\boldsymbol{B})$ and for 14 datasets of three bipolar neurons (C). D, Shows distributions of first-spike latency (bin width $1 \mathrm{~ms}$ ) for neurons classified as bipolar (white, solid line) and monopolar (dark gray, dashed line). $\boldsymbol{E}$, Shows PSTHs, normalized per neuron (see Materials and Methods). Note the close correspondence between first-spike latency distributions (D) and the PSTHs (E). a.u., arbitrary unit.
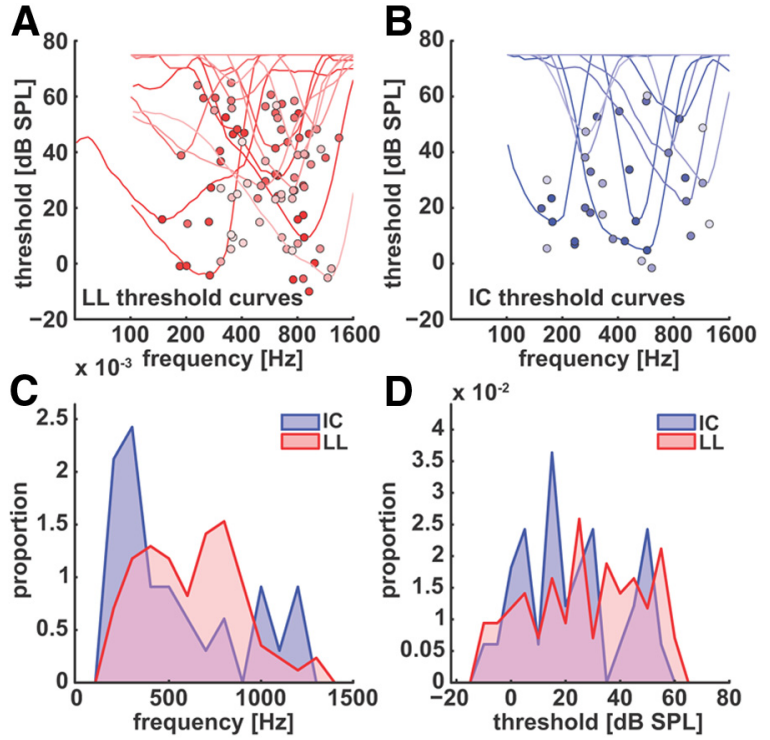

Figure 6. Frequency tuning curves (FTCs) obtained from the $\mathrm{LL}(\boldsymbol{A})$ and $\mathrm{IC}(\boldsymbol{B})$. For visual purposes only a subset of the data ( 12 of $85 \mathrm{LL}$ and 9 of $33 \mathrm{IC}$ ) are shown as curves (5 point running average). The horizontal segments near $80 \mathrm{~dB}$ reflect the ceiling SPL of the threshold tracking algorithm. CF and threshold of all neurons recorded are indicated with filled circles. Differences in shading help to visually distinguish different neurons. $\boldsymbol{C}$, Distributions of CF of the LL (red) and the IC (blue) with a bin width of $100 \mathrm{~Hz}$. Note that the IC data are biased toward CFs smaller than $500 \mathrm{~Hz}$. D, Probability distributions of response thresholds for the LL (red) and the IC (blue) with a bin width of $5 \mathrm{~dB}$ SPL. countered a large number of low-frequency units both in the LL and in the IC. The IC distribution is clearly skewed toward units with $\mathrm{CFs}<500 \mathrm{~Hz}$ while the LL distribution shows a broader peak $<1000 \mathrm{~Hz}$. Certainly for the IC, the placement of the electrodes biased the sample toward low CFs. To some extent, for both the IC and the LL, the binaural beat search stimulus may have biased the CF distribution, but note that the search stimulus extended up to $20 \mathrm{kHz}$, so that the low-CF bias in the LL is stronger than expected. The threshold distributions of the LL and the IC (Fig. 6D) are very similar. They span a range from approximately $-10 \mathrm{~dB}$ SPL up to $\sim 60 \mathrm{~dB}$ SPL. The very low thresholds at low CFs may be exaggerated due to the opening of the bullas (Rosowski et al., 2006). We compared the $\mathrm{Q}_{10}$ values as a function of $\mathrm{CF}$ with the trend line reported for chinchilla auditory nerve (AN) (Ruggero and Temchin, 2005), and found good agreement (data not shown). $\mathrm{Q}_{10}$ percentiles $(25 / 50 / 75)$ were as follows: $\mathrm{LL}_{\mathrm{Q} 10}: 1.00 / 1.38 / 2.21 ; \mathrm{IC}_{\mathrm{Q} 10}: 1.02 / 1.79 / 2.43$.

The abundance of low-frequency neurons and their sharp and sensitive tuning renders the chinchilla an interesting species for binaural physiology. Next we characterize ITD-sensitivity probed with binaural beat stimuli.

\section{Responses to binaural beats}

Figure $7 A$ shows a dot raster plot of a LL recording $(\mathrm{CF}$ of $308 \mathrm{~Hz})$ in response to a $2 \mathrm{~Hz}$ binaural beat of varying $f_{\text {car }}$. Poststimulus time and $f_{\text {car }}$ are plotted on the bottom abscissa and the ordinate, respectively. Dots indicate the occurrence of a spike, the vertical dashed line marks stimulus offset. Spike rate (top abscissa) is shown as a black line with circular markers: it peaks at $f_{\text {car }}$ s close 


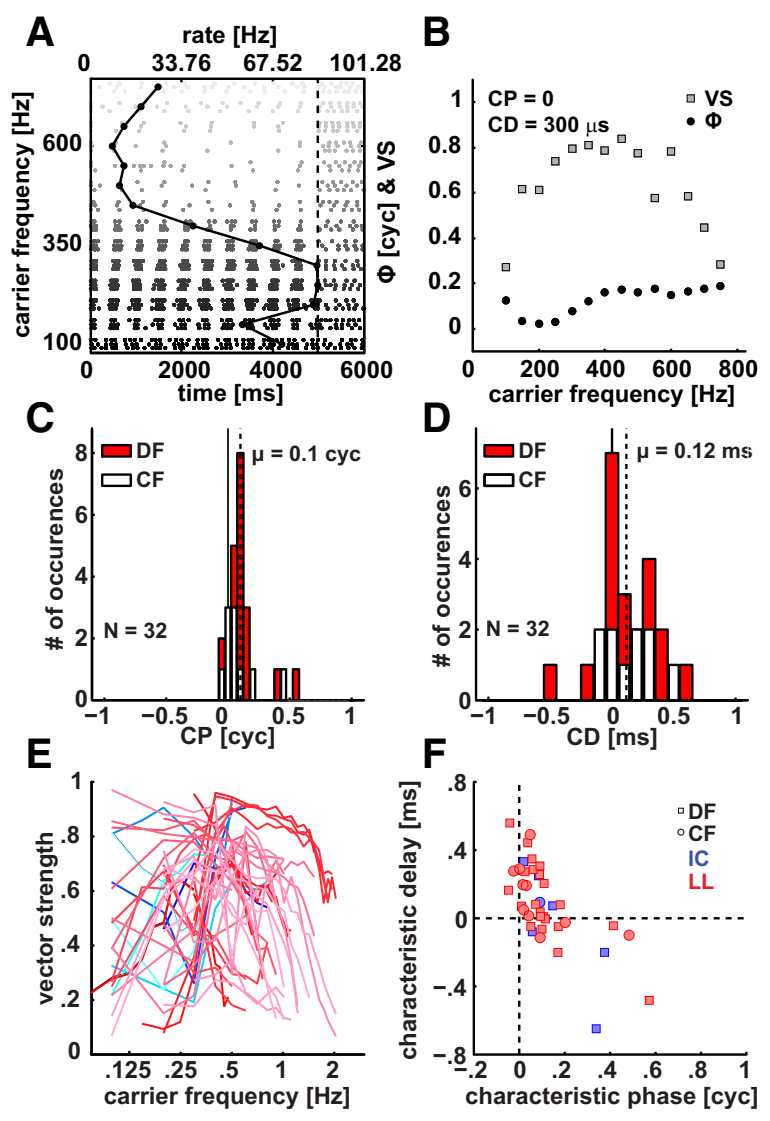

Figure 7. Binaural beat analysis. $\boldsymbol{A}$, Dot raster plot and spike rate (solid black line with symbols) as a function of carrier frequency ( $\left.f_{\text {car }}\right)$ for an example neuron with monopolar spike waveform. Vertical dashed line indicates stimulus duration. Spikes (dots) cluster at a certain phase of $f_{\text {beat }}$ and the spike rate is maximal close to the $\mathrm{CF}_{\text {of }} 308 \mathrm{~Hz}$. $\boldsymbol{B}$, Interaural phase $\varphi$ (black circles) and vector strength (VS) (gray squares) as a function of $f_{\text {car }}$. The black line indicates a linear fit to the phase data. The intercept with the ordinate is the characteristic phase (CP) and the slope is the characteristic delay (CD). CP and CD distributions of a population of 32 individual $\mathrm{LL}$ neurons are shown in subplot of $\boldsymbol{C}$ and $\boldsymbol{D}$, respectively. The dashed lines indicate the circular mean $(\boldsymbol{C})$ and the mean $(\boldsymbol{D})$ of the distributions. Red and white boxes indicate units for which only a dominant frequency (DF) measurement or the CF were available, respectively. $A C D$ of 0 $\mathrm{ms}$ is indicated by the vertical solid black line. $\boldsymbol{E}, \boldsymbol{F}$, Population data for interaural phase difference (IPD) sensitivity to binaural beat stimuli. Blues, IC responses $(N=7)$; reds, LL responses $(N=32)$. $E$, Magnitude of IPD sensitivity, measured with VS to $f_{\text {beat }}(=2 \mathrm{~Hz})$ as a function of $f_{\text {car }}, F, C D-C P$ relationship. Neurons for which the CF or only the DF was available are indicated with circles and squares, respectively.

to the unit's CF of $308 \mathrm{~Hz}$. Furthermore, it is apparent that the responses at most $f_{\text {car }} \mathrm{s}$ are clustered around a specific phase of the beat frequency. This phase is somewhat later in the IPD cycle for increasing $f_{\text {car }}$, visible as a gentle tilt to the right in the dot rasters.

To quantify this behavior we performed a standard binaural beat analysis (Yin and Kuwada, 1983) and plotted VS and mean $\operatorname{IPD}(\varphi)$ as a function of carrier frequency in Figure $7 B$. We excluded all points that according to the Rayleigh test were not significantly phase locked. For this example VS (light gray squares) peaks over a broad range of $f_{\text {car }}$ centered near $400 \mathrm{~Hz}$. In this frequency range VS values are close to 0.8 indicating strong IPD sensitivity. Note that, with decreasing $f_{\text {car }}$, the VS values decrease from this maximum, even though firing rate (Fig. 7A) is still high. We will return to this point below (Fig. 7E). With increasing $f_{\text {car }}$ phase accumulates reaching a maximum of 0.2 cycles at $750 \mathrm{~Hz}$. A linear fit through the $\varphi$ versus $f_{\text {car }}$ relation yields an intercept, i.e., a CP, of 0 cycles and a slope, i.e., a CD, of $300 \mu \mathrm{s}$. These are typical values for our population as can be seen in Figure $7 C$ and $D$. The histogram in Figure $7 C$ shows the $C P$ distribution of our sample of 32 neurons recorded from the LL. We have split the dataset in neurons for which only the DF (red) was available and those for which we obtained the CF (white). The circular mean of CP across DF and CF of 0.1 cycles falls close to 0 cycles; values larger than 0.2 are rare. This indicates that most units were of the peak type. This dominance of peak-type units (CP close to 0 ) is consistent with the assumption that most of our recordings stem from the MSO. In contrast, LSO neurons, due to their excitatory/inhibitory interactions, exhibit trough-type characteristics with CPs close to 0.5 (Joris, 1996; Batra et al., 1997; Tollin and Yin, 2005). Interestingly, there is a small cluster of three neurons that had a CP close to 0.5 . Here it should be noted that not only LSO but also MSO neurons have been reported to exhibit CPs close to 0.5 (gerbil: Day and Semple, 2011). Testing for ILD sensitivity would lend further support for an LSO versus MSO origin but was not performed due to constraints in the time of recording. The $\mathrm{CD}$ distribution (Fig. $7 D$ ) is centered near zero ITD. With a value of $120 \mu \mathrm{s}$, the mean even falls within the ethological range of $\sim 300 \mu$ s of the chinchilla (Koka et al., 2011).

A question of interest (Joris and Verschooten, 2013) is the upper frequency limit at which sensitivity to binaural beats is obtained, as this type of sensitivity stands as a model for the use of temporal fine-structure by the central auditory system. Figure $7 E$ shows VS values as a function of stimulus frequency for our population of IC (blue) and LL (red) neurons. Only significant values are shown (see Materials and Methods). Significant IPD sensitivity is found up to $2 \mathrm{kHz}$. In the IC this was the highest $f_{\text {car }}$ tested, so we cannot be sure it is an upper limit, but it is the same limit as reported for auditory cortex (Benson and Teas, 1976) and in the LL one neuron was tested up to $f_{\text {car }}$ of $3 \mathrm{kHz}$ but was only IPD sensitive up to $2 \mathrm{kHz}$. We therefore surmise that $2 \mathrm{kHz}$ is the upper limit of IPD sensitivity or is very close to it. A second feature of note is the sharp drop-off of IPD sensitivity at low frequencies in many neurons, giving many IPD functions a bandpass character. This is particularly the case for stimulus frequencies $<\sim 400 \mathrm{~Hz}$. Examination of the IPD functions at these frequencies shows no evidence for peak splitting, but rather the appearance of a sustained response without any stimulus-related periodicity. This is visible in Figure $7 A$ : the neuron is driven at similar spike rates at 100 and $350 \mathrm{~Hz}$ (Fig. 7A, solid line), but IPD sensitivity (Fig. 7B; VS values to $f_{\text {beat }}$ ) is poor at the lowest frequency.

Figure $7 F$ shows an inverse relationship between $\mathrm{CD}$ and $\mathrm{CP}$ for a combined population of IC and LL neurons. Such an inverse relationship was also described in the IC of guinea pig and cat (McAlpine et al., 1996; Joris and Karino, 2012) and in the DNLL of the guinea pig and gerbil (Agapiou and McAlpine, 2008; Lüling et al., 2011). The relationship in Figure $7 F$ is very similar to that in guinea pig, while it is less tight in cat and gerbil. The origin and significance of the relationship are unclear.

As described in the Materials and Methods section we also constructed TDFs and composite curves from the binaural beat data to extract $\mathrm{BD}$ values. The contour plot in Figure $8 A$ gives an overview of the TDFs for the example unit of Figure $7 A$ and $B$. ITD and $f_{\text {car }}$ are the ordinate and abscissa, respectively. Spike rate has been normalized to the maximal rate so that white and black contours correspond to a value of one and zero, respectively. The white solid and dashed hyperbolic lines indicate the extent of a stimulus cycle and $1 / 4$ of a stimulus cycle. The upper white line, referred to as the $\pi$-limit because it is separated by half a stimulus cycle from 0 ITD, describes an upper bound that is found empirically in the distribution of CD or BD in different species (Benson 

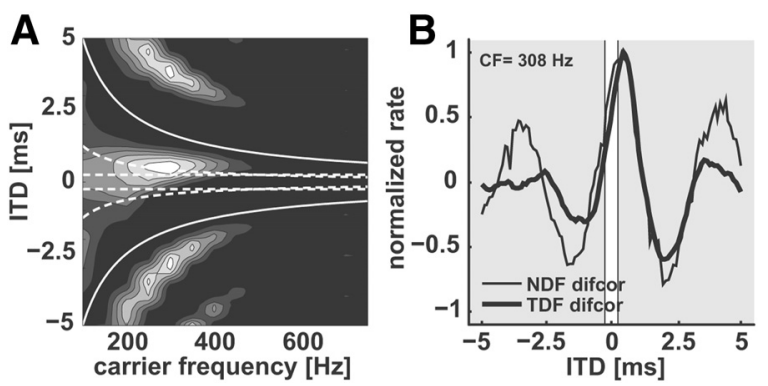

Figure 8. A Contour plot of tone delay functions (TDFs) as a function of $f_{\text {car }}$ constructed from binaural beat data for the neuron also illustrated in Figure $7 A$ and $B$. Spike rate has been normalized so that white indicates a value of 1 and black a value of 0 . Dashed white horizontal lines indicate the approximate ethological ITD range of the chinchilla. White hyperbolic solid and dashed lines indicate the $\pi$-limit and $1 / 4 \pi$-limit, respectively. $\boldsymbol{B}$, Difcor of responses to binaural beat data. The white patch indicates the approximate ethological ITD range. For comparison, the difcor obtained from noise delay functions (NDFs) of the same neuron is shown as a thin line. For this neuron, there is good agreement between the BD obtained from the composite tonal (TDF difcor) and noise (NDF difcor) data.

and Teas, 1976; McAlpine et al., 1996; Brand et al., 2002; Hancock and Delgutte, 2004; Joris et al., 2006; Pecka et al., 2008). A streak of high spike rates is seen contralaterally close to zero milliseconds but outside the ethological ITD range (indicated with dashed white, horizontal lines). As expected peak activity is reached close to the unit's CF of $\sim 300 \mathrm{~Hz}$ at an ITD of $\sim 300 \mu \mathrm{s}$. Activity outside the $\pi$-limit indicates side peaks in the TDFs. The side peaks, necessarily, follow a hyperbolic trajectory and the distance between a given side peak and the main peak near to zero ITD corresponds to the stimulus period. Collapsing the data along the frequency axis onto the ITD axis, i.e., summing all TDFs and normalizing the sum to the resulting maximum, yields the so-called composite curve (Yin et al., 1986), which gives a reasonable prediction of the response to correlated broadband noise. We computed the composite curve and also the corresponding prediction for the response to anti-correlated noise, following the procedure of Joris et al. (2005). Subtraction of the two curves gives the difcor shown in Figure $8 B$ as a thick black line. The BD, again defined as the ITD of maximal response, is positive (i.e., in response to a stimulus leading in the contralateral ear) close to zero ITD but outside of the ethological range. The side peaks of the composite curve are suppressed relative to the side peaks of the TDFs. This is because they do not line up across frequencies (Fig. 8A). Together Figure $8, A$ and $B$, demonstrates the procedure used to obtain BDs from binaural beat data. We extracted BDs for all of our units from the simulated difcors. Before discussing these data we first present typical generic NDFs in the next section.

\section{NDFs}

Along with the difcors to tones (thick line), Figure $8 B$ also shows the difcor obtained from actual NDFs (thin line). The derivation of such difcors is illustrated in Figure 9, for two LL units $(A, B)$ and one IC unit $(C)$ with similar low CFs $(200-300 \mathrm{~Hz})$. Shown are the responses to correlated ( $\rho=1$; blue) and anti-correlated ( $\rho=-1$; red) Gaussian noise bursts of varying ITD, as well as their subtraction (black). The error bars indicate the SD of the responses. As expected for low-CF neurons, the NDF to anticorrelated noise approximates a mirror image of that to correlated noise, i.e., peaks and troughs were inverted, indicating that the ITD sensitivity to noise is dominated by fine-structure (Joris, 2003).
To compare spike rates in ITD-sensitive IC (blue) and LL (red) neurons, we constructed histograms (Fig. 10) of driven rates at $\mathrm{BD}(A)$, nondriven rate ( $B$; i.e., rate after stimulus offset), and modulation depth $\left(\left(R_{\max }-R_{\min }\right) / R_{\max }\right)$. We used both NDF data and composite curves derived from binaural beat data. LL neurons tended to have higher maximal ( $p \ll 0.01, \alpha=0.01$; Kolmogorov-Smirnov test) and nondriven rates $(p=0.004, \alpha=$ 0.01 ; Kolmogorov-Smirnov test) than IC neurons, but modulation depth was very similar for the two populations $(p=0.92$, $\alpha=0.01$; Kolmogorov-Smirnov test).

\section{BD distributions}

Finally, in Figure 11 we present BD versus CF or DF for neurons recorded in the LL (red) and in the IC (blue). BDs are shown separately for NDFs $(A)$ and composite curves $(B)$, as well as combined $(C)$. The abscissa indicates either CF (circles) or DF (inverted triangles). If multiple datasets for one neuron were available, we averaged the BDs. The solid and dashed hyperbolic lines represent the $\pi$-limit and the $1 / 4 \pi$-limit, respectively. As already remarked (Fig. 6), the distribution of IC units is biased toward lower CFs compared with the LL.

Several features of the BD distribution are consistent with previous reports in $\mathrm{MSO}$ and IC. Most BDs are on the positive half of the ordinate indicating a preference for contralaterally leading binaural stimuli. Furthermore, there is a clear dependence of the range of BDs on CF: the range and mean value are higher at low CF than at high CF. This is particularly clear for the BDs obtained to noise (Fig. 11A).

However, the distribution also differs from most previous reports in rodents in two ways. A first interesting observation is that the majority of BDs in the LL (65\%) and a large fraction in the IC $(41 \%)$ are within the ethological ITD range. These numbers are approximate as the exact ITD range is dependent on head size and frequency, where larger heads and lower frequencies generate a larger ITD range. For example, in the chinchilla the ITD range is $\sim 250 \mu$ s at $1 \mathrm{kHz}$ (Fig. 11, white patch) and $\sim 300 \mu$ s at $250 \mathrm{~Hz}$ (Fig. 11, dark gray patch; Koka et al., 2011), and at lower frequencies the ITD range is not known but may be even larger. Note that for almost $60 \%$ of the data shown the abscissa indicates CF; for the remainder, $\mathrm{DF}$ was used $\left(\mathrm{CF}_{\mathrm{LL}}: 13\right.$ and $\mathrm{DF}_{\mathrm{LL}}: 15 ; \mathrm{CF}_{\mathrm{IC}}: 16$ and $\left.\mathrm{DF}_{\mathrm{IC}}: 6\right)$.

A second and related observation is that small BDs are also found at low CFs and that they are rather widely distributed between the $\pi$-limit and the horizontal line at ITD $=0 \mathrm{~ms}$. This is in stark contrast to reports from other rodents (gerbil MSO: Brand et al., 2002; DNLL: Siveke et al., 2006; guinea pig DNLL and IC: Agapiou and McAlpine, 2008; McAlpine et al., 1996, 2001), in which BDs are less dispersed and cluster around the $1 / 4$ $\pi$-limit. In this regard, the present data seem to be in better agreement with data from cats (Hancock and Delgutte, 2004; Joris et al., 2006) and a report in gerbil (Day and Semple, 2011).

In the physiological ITD literature, various measures for "optimal delay" have been used (see Discussion). For neurons for which BD estimates from both NDFs (responses to noise) and composite curves (responses to binaural beats) were available, we compared the two (data not shown): the correlation was poor $\left(R^{2}=0.35, p=0.2, N=15\right)$.

\section{Discussion}

The circuits that generate ITD sensitivity are well established as a model system to study temporal processing, but characterization of the primary binaural comparison has suffered from the difficulty in obtaining good spike isolation with extracellular record- 

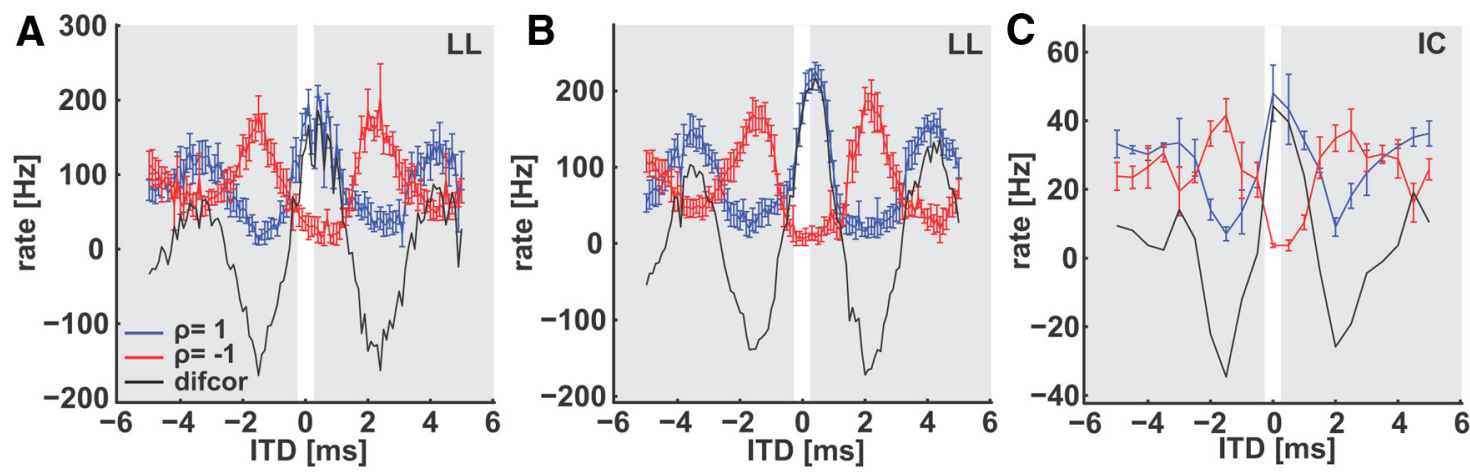

Figure 9. NDFs to correlated (blue) and anti-correlated (red) noise bursts for two example LL neurons ( $\boldsymbol{A}, \mathrm{CF}=172 \mathrm{~Hz} ; \boldsymbol{B}, \mathrm{CF}=308 \mathrm{~Hz}$; both monopolar spike waveforms) and one IC neuron (C, (F $200 \mathrm{~Hz}$ ) neuron. The difcor, i.e., the difference between correlated and anti-correlated NDFs, is shown as a black line. Error bars indicate the SD over three repetitions and the white patch shows the approximate ethological ITD range. Stimulus parameters for the Gaussian noise burst were bandwidth $0.05-8 \mathrm{kHz}$ presented for $1 \mathrm{~s}$ at $70 \mathrm{~dB}$ SPL with an interstimulus interval of $1.1 \mathrm{~s}$ and repeated three times. Data in $\boldsymbol{B}$ are for same neuron as in Figure $7 A$ and $B$ and 8 .
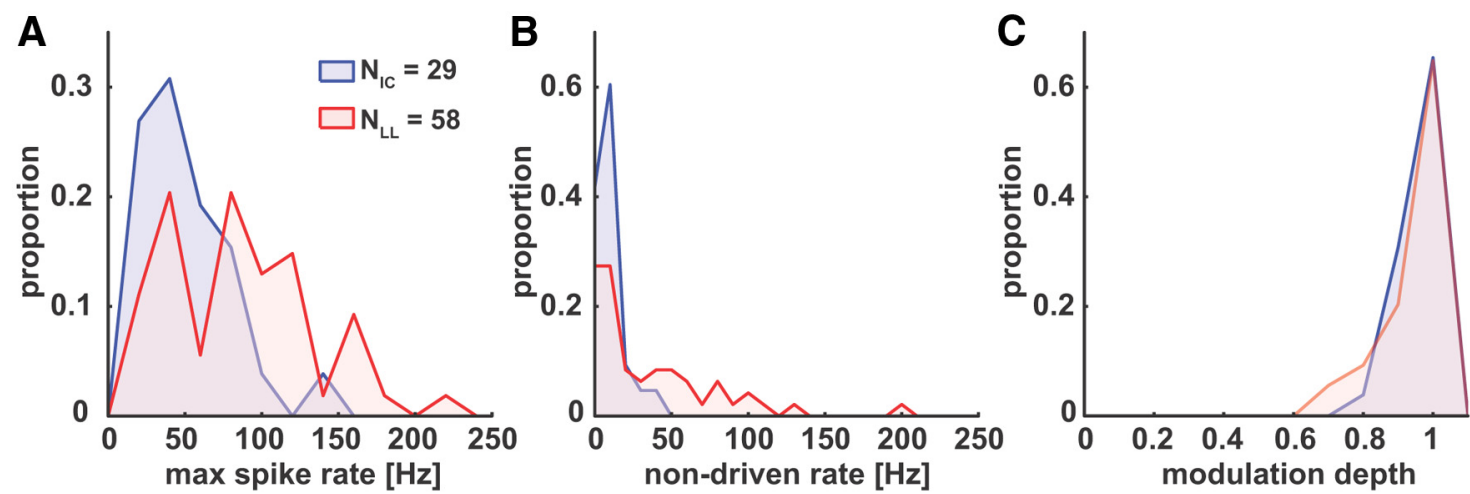

Figure 10. Histograms of spike rate at $B D(\boldsymbol{A})$, nondriven rate $(\boldsymbol{B})$, and modulation depth $(\boldsymbol{C})$ for $\mathrm{IC}($ blue $)$ and $\mathrm{LL}($ red $)$ neurons obtained with NDFs and from composite curves. Modulation depth $=$ $\left(R_{\max }-R_{\min }\right) / R_{\max }$

ings (Irvine, 1986). This difficulty limits not only the number of neurons reported, but also the quality and variety of the recordings. The data available are mostly responses to tones (but see Rupert et al., 1966; Yin and Chan, 1990): little is known regarding ITD tuning to broadband stimuli such as noise or clicks, or even regarding very basic properties such as frequency tuning. Recent in vitro studies suggest that the recording difficulty is tied to specialized cellular properties of MSO neurons toward temporal computation (Scott et al., 2010; Golding and Oertel, 2012). Other recording strategies are thus needed. Juxtacellular (van der Heijden et al., 2013) and whole-cell (Franken et al., 2013) recordings are a promising avenue to address the cellular mechanisms of ITD sensitivity in vivo, but their feasibility may be restricted to small species with a superficially located MSO. The trapezoid body-a heavily myelinated bundle of fibers also containing MSO afferents-overlies the MSO and hampers whole-cell recordings in larger species. Here we took an alternative approach in which heavy myelination is actually a bonus: we used sharp micropipettes to record from MSO axons in the LL of the chinchilla. The histological analysis of electrode tracks marked with a fluorescent dye showed that we recorded at the level of the DNLL or dorsal to it (Figs. 1, 2). Consistent with previous experience in regions with myelinated bundles and associated nuclei (SOC: Smith et al., 1993; dorsal and intermediate acoustic strias: Joris and Smith, 1998; LL: P. H. Smith and P. X. Joris, unpublished observations), these recordings are dominated by monopolar spike waveforms. Bipolar spike waveforms occurred only occasionally and were associated with a drop in electrode impedance, likely due to tip damage. Importantly, the latter recordings yielded responses with longer latency $(10-15 \mathrm{~ms})$ than the monopolar recordings ( $\sim 7 \mathrm{~ms}$; Fig. 5). Moreover the latency distribution of monopolar recordings was unimodal and not bimodal as would be expected if they also sampled DNLL axons. As suggested by a small cluster of $\mathrm{CP}$ values near 0.5 (Fig. $7 \mathrm{C}$ ), there may be a contribution of low-CF LSO axons. Although VNLL neurons show a form of ITD sensitivity (Batra and Fitzpatrick, 2002), it is very different from the sustained ITD sensitivity shown here so that these neurons are not likely to contribute to our database. Ultimately, intra-axonal labeling will be required to firmly establish the MSO origin of the recordings.

The main difficulty encountered was poor mechanical stability. The recordings lasted up to $1 \mathrm{~h}$ per fiber and routinely $\sim 30$ min. FTCs were routinely obtained (Fig. 6), as well as highquality recordings to broadband noise (Figs. 4, 9). It is interesting to observe that the spikes recorded were stable in size for a given recording (Fig. 4), unlike the graded action potentials recorded somatically (Scott et al., 2010). A surprising finding is that the recordings were strongly biased to low-frequency and IPDsensitive neurons. A spatial sampling bias is unlikely given that we used two, spatially almost orthogonal, approaches. Although the search stimulus used (tone pips and binaural beats) may induce some bias, we surmise that the main source must be in the size of the axons. We are not aware of anatomical studies of axon caliber in LL: our prediction is that MSO axons are larger than fibers from CN, LLN, or other SOC nuclei. Besides the abundance of low-CF binaural neurons, low thresholds and clear frequency 
A

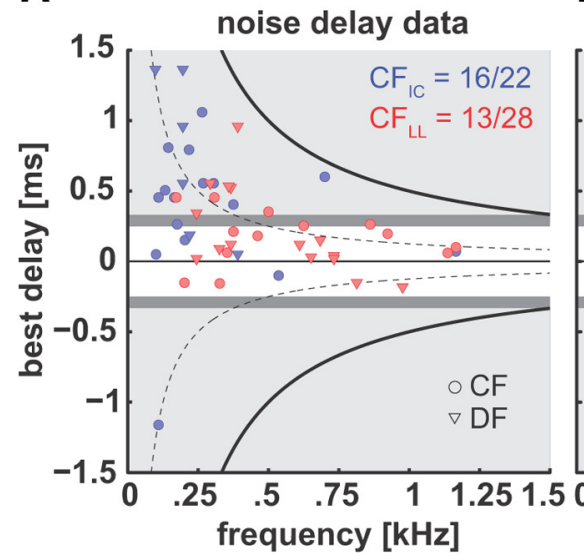

B

binaural beat data

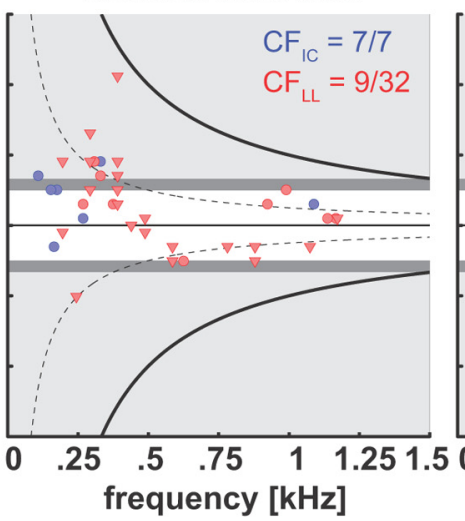

C noise delay \& binaural beat data

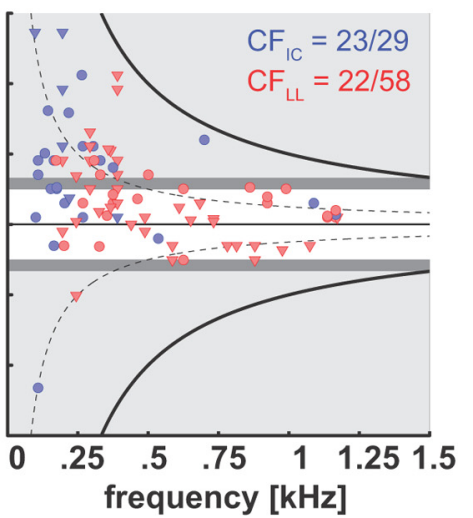

Figure 11. BD obtained from NDFs ( $\boldsymbol{A}$ ), composite curves $(\boldsymbol{B})$, and both together ( $\boldsymbol{C}$ ) for all LL (red) and IC (blue) data. The BD is graphed as a function of CF (circles) or DF (inverted triangles). Each symbol represents one neuron. The white and the dark gray patches show the approximate ethological ITD ranges for high ( $1 \mathrm{kHz}$, smallest range) and low ( $250 \mathrm{~Hz}$, largest range) frequencies, and the black hyperbolic solid and dashed lines indicate the $\pi$-limit and $1 / 4 \pi$-limit, respectively. The legend entry $\mathrm{CF}_{\mathrm{XX}}$ followed by two numbers indicates the number of neurons in the IC and LL for which CFs where available (first number) and the total number of neurons (second number). The difference between these two values is the number of neurons for which we did not obtain a CF but used the DF instead.

tuning (Fig. 6) contribute to the appeal of this experimental model.

Since data on the binaural sensitivity of the chinchilla are limited (Benson and Teas, 1976; Chen and Sinex, 1999) we also collected data from the IC. At the level of analysis presented here, we did not see striking differences between IC and LL. Spike rates tend to be somewhat larger in LL than in IC (Fig. 10A,B). Frequency tuning was similar, with sensitive and well tuned low-CF neurons present in both LL and IC (Fig. 6). There were no obvious differences in the shape of ITD tuning curves (Fig. 9) or in the BD distributions (Fig. 11). Note that the distributions of CFs sampled were somewhat different in the two populations (Fig. $6 C$ ). These observations lend credit to the general assumption that binaural properties at the level of the IC largely reflect the properties created at the level of the SOC, but again more extensive analyses along more stimulus and response dimensions and on larger samples are needed to better appreciate any differences between the two structures.

An unexpected and quite striking observation in the chinchilla is the frequent lack of IPD sensitivity at very low frequencies, both in LL and IC (Fig. 7E), despite low thresholds (Fig. 6) and the presence of driven responses (Fig. 7A). The fact that, at these very low frequencies, afferents show good phase locking (auditory nerve: Temchin and Ruggero, 2010, bushy cells: Recio-Spinoso, 2012), and that there is good IPD sensitivity in at least some LL axons and IC neurons, suggests that the limiting factor is at the level of (some neurons of) the MSO itself. We have not explored the phenomenon but it is worth further study as it affects the interpretation of the presence of low-frequency hearing and sound localization ability (Heffner et al., 1994) enabled by large bullas (Webster and Plassmann, 1992) and may give further insight in the process of coincidence detection.

A relation between $\mathrm{BD}$ and $\mathrm{CF}$ was reported for the guinea pig IC by McAlpine et al. (1996, 2001). Remarkably, BDs respect the so-called $\pi$-limit, which corresponds to half a cycle of the CF. This relationship has received much attention as it is unpredicted by a pure axonal time delay model (Jeffress, 1948). Moreover, the BDs in guinea pig cluster around a value of $\pi / 4$, and are outside of the approximate ethological range of ITDs at the lowest CFs. This observation has led to a revival of "hemispheric" models (Von
Békésy, 1960; van Bergeijk, 1962; Hall, 1965), which propose that the code for azimuthal location is in a comparison of overall activity in left and right hemispheres rather than in a topographically mapped array of neurons with different CDs (Jeffress, 1948; Licklider, 1959). In our data there is no obvious clustering of BDs around a value of $\pi / 4$, and there is an abundance of BDs close to zero $\mathrm{ms}$ and within the ethological ITD-range (Fig. 11). In fact the distribution is not very different from that reported in cat (Hancock and Delgutte, 2004; Joris et al., 2006), though the chinchilla seems to have even more neurons tuned to small BDs, which fall inside its approximate ethological ITD range of $\sim 250$ $\mu$ s (Koka et al., 2011; Lupo et al., 2011), even for CFs $<500 \mathrm{~Hz}$. This is rather unlike the guinea pig, where hardly any BDs are inside that range for CFs $<300 \mathrm{~Hz}$ (McAlpine, 2005). This seems to be a genuine species difference or may reflect sampling biases in either species.

In our own view, the BD-CF relationship forms an important constraint regarding the nature of the mechanism(s) generating the $\mathrm{BD}$, but is not very constraining regarding the nature of ITD coding. It is noteworthy that an early study performed in auditory cortex of the chinchilla (Benson and Teas, 1976) also reported a BD-CF relation within the $\pi$-limit, from which it also favored a hemispheric coding scheme. However, the BDs reported were for pure tones at BF, which by necessity show a hyperbolic pattern when plotted on a time rather than a phase axis (cf. Reale and Brugge, 1990). Multiple metrics have been used to express the optimal delay (Benson and Teas, 1976) of ITD tuning. The CD to tones, $\mathrm{BD}$ to noise, $\mathrm{BD}$ of the composite curve calculated from tones, and best ITDs of single tones, are all instructive metrics, but they can differ substantially, which hampers comparisons across studies. Similarly, different measures have been used to express frequency tuning. BF, defined as the frequency giving maximal spike rate, can differ substantially from CF both in binaural neurons and their monaural inputs (Joris et al., 1994a,b). Thus, the choice of metrics for both the abscissa and ordinate can substantially affect distributions as in Figure 11.

How these "technical" issues affect conclusions regarding coding has not been adequately dealt with in the binaural literature and needs to be sorted out. The LL approach developed here opens a new window to the MSO that can contribute to resolving 
these issues by allowing a wider range of stimuli and a more thorough characterization of binaural properties than has been possible with other recording techniques.

\section{References}

Agapiou JP, McAlpine D (2008) Low-frequency envelope sensitivity produces asymmetric binaural tuning curves. J Neurophysiol 100:2381-2396. CrossRef Medline

Batra R, Fitzpatrick DC (2002) Processing of interaural temporal disparities in the medial division of the ventral nucleus of the lateral lemniscus. J Neurophysiol 88:666-675. Medline

Batra R, Kuwada S, Fitzpatrick DC (1997) Sensitivity to interaural temporal disparities of low- and high-frequency neurons in the superior olivary complex. I. Heterogeneity of responses. J Neurophysiol 78:1222-1236. Medline

Benson DA, Teas DC (1976) Single unit study of binaural interaction in the auditory cortex of the chinchilla. Brain Res 103:313-338. CrossRef Medline

Brand A, Behrend O, Marquardt T, McAlpine D, Grothe B (2002) Precise inhibition is essential for microsecond interaural time difference coding. Nature 417:543-547. CrossRef Medline

Brugge JF, Anderson DJ, Aitkin LM (1970) Responses of neurons in the dorsal nucleus of the lateral lemniscus of cat to binaural tonal stimulation. J Neurophysiol 33:441-458. Medline

Chen GD, Sinex DG (1999) Effects of interaural time differences on the responses of chinchilla inferior colliculus neurons to consonant-vowel syllables. Hear Res 138:29-44. CrossRef Medline

Coffey CS, Ebert CS Jr, Marshall AF, Skaggs JD, Falk SE, Crocker WD, Pearson JM, Fitzpatrick DC (2006) Detection of interaural correlation by neurons in the superior olivary complex, inferior colliculus and auditory cortex of the unanesthetized rabbit. Hear Res 221:1-16. CrossRef Medline

Day ML, Semple MN (2011) Frequency-dependent interaural delays in the medial superior olive: implications for interaural cochlear delays. J Neurophysiol 106:1985-1999. CrossRef Medline

DiCarlo JJ, Lane JW, Hsiao SS, Johnson KO (1996) Marking microelectrode penetrations with fluorescent dyes. J Neurosci Methods 64:75-81. CrossRef Medline

Franken TP, Roberts MT, Wei L, Golding NL, Joris PX (2013) In vivo whole-cell recordings from principal neurons of the medial superior olive. Assoc Res Otolaryngol Abs 36:353.

Goldberg JM, Brown PB (1969) Response of binaural neurons of dog superior olivary complex to dichotic tonal stimuli. J Neurophysiol 32:613636. Medline

Golding NL, Oertel D (2012) Synaptic integration in dendrites: exceptional need for speed. J Physiol 590:5563-5569. CrossRef Medline

Grothe B (2003) New roles for synaptic inhibition in sound localization. Nat Rev Neurosci 4:540-550. CrossRef Medline

Guinan, JJ, Guinan SS, Norris BE (1972) Single auditory units in the superior olivary complex I: responses to sounds and classifications based on physiological properties. Intern J Neurosci 4:101-120. CrossRef

Hall JL 2nd (1965) Binaural interaction in the accessory superior-olivary nucleus of the cat. J Acoust Soc Am 37:814-823. CrossRef Medline

Hancock KE, Delgutte B (2004) A physiological model of interaural time difference discrimination. J Neurosci 24:7110-7117. CrossRef Medline

Heffner RS, Heffner HE, Kearns D, Vogel J, Koay G (1994) Sound localization in chinchillas. I: left/right discriminations. Hear Res 80:247-257. CrossRef Medline

Helfert RH, Bonneau JM, Wenthold RJ, Altschuler RA (1989) GABA and glycine immunoreactivity in the guinea pig superior olivary complex. Brain Res 501:269-286. CrossRef Medline

Irvine DRF (1986) The auditory brainstem: a review of the structure and function of auditory brainstem processing mechanisms. Berlin: Springer.

Jeffress LA (1948) A place theory of sound localization. J Comp Physiol Psychol 41:35-39. CrossRef Medline

Jercog PE, Svirskis G, Kotak VC, Sanes DH, Rinzel J (2010) Asymmetric excitatory synaptic dynamics underlie interaural time difference processing in the auditory system. PLoS Biol 8:e1000406. CrossRef Medline

Joris PX (1996) Envelope coding in the lateral superior olive. II. Characteristic delays and comparison with responses in the medial superior olive. J Neurophysiol 76:2137-2156. Medline

Joris PX (2003) Interaural time sensitivity dominated by cochlea-induced envelope patterns. J Neurosci 23:6345-6350. Medline
Joris PX, Karino S (2012) Relationship between binaural characteristic delay and phase is consistent with cochlear disparities. Assoc Res Otolaryngol Abs 35:73.

Joris PX, Smith PH (1998) Temporal and binaural properties in dorsal cochlear nucleus and its output tract. J Neurosci 18:10157-10170. Medline

Joris PX, Verschooten E (2013) On the limit of neural phase locking to fine-structure in humans. In: Basic aspects of hearing: physiology and perception. New York: Springer.

Joris PX, Yin TC (1995) Envelope coding in the lateral superior olive. I. Sensitivity to interaural time differences. J Neurophysiol 73:1043-1062. Medline

Joris PX, Carney LH, Smith PH, Yin TC (1994a) Enhancement of neural synchronization in the anteroventral cochlear nucleus. I. Responses to tones at the characteristic frequency. J Neurophysiol 71:1022-1036. Medline

Joris PX, Smith PH, Yin TC (1994b) Enhancement of neural synchronization in the anteroventral cochlear nucleus. II. Responses in the tuning curve tail. J Neurophysiol 71:1037-1051. Medline

Joris PX, Van De Sande, van der Heijden M (2005) Temporal damping in response to broadband noise. I. Inferior colliculus. J Neurophysiol 93: 1857-1870. Medline

Joris PX, Van de Sande B, Louage DH, van der Heijden M (2006) Binaural and cochlear disparities. Proc Natl Acad Sci U S A 103:12917-12922. CrossRef Medline

Koka K, Jones HG, Thornton JL, Lupo JE, Tollin DJ (2011) Sound pressure transformations by the head and pinnae of the adult chinchilla (Chinchilla lanigera). Hear Res 272:135-147. CrossRef Medline

Kuwada S, Yin TC, Wickesberg RE (1979) Response of cat inferior colliculus neurons to binaural beat stimuli: possible mechanisms for sound localization. Science 206:586-588. CrossRef Medline

Kuwada S, Stanford TR, Batra R (1987) Interaural phase-sensitive units in the inferior colliculus of the unanesthetized rabbit: effects of changing frequency. J Neurophysiol 57:1338-1360. Medline

Kuwada S, Batra R, Stanford TR (1989) Monaural and binaural response properties of neurons in the inferior colliculus of the rabbit: effect of sodium pentobarbital. J Neurophysiol 61:269-282. Medline

Licklider JCR (1959) Three auditory theories. In: Psychology: a study of a science. Study I. Conceptual and systematic. Vol. 1. Sensory, perceptual, and physiological formulat, 41-144. New York: McGraw-Hill.

Louage DH, van der Heijden M, Joris PX (2004) Temporal properties of responses to broadband noise in the auditory nerve. J Neurophysiol 91: 2051-2065. CrossRef Medline

Louage DH, van der Heijden M, Joris PX (2005) Enhanced temporal response properties of anteroventral cochlear nucleus neurons to broadband noise. J Neurosci 25:1560-1570. CrossRef Medline

Lüling H, Siveke I, Grothe B, Leibold C (2011) Frequency-invariant representation of interaural time differences in mammals. PLoS Comput Biol 7:e1002013. CrossRef Medline

Lupo JE, Koka K, Thornton JL, Tollin DJ (2011) The effects of experimentally induced hearing loss on spectral and temporal aspects of sound transmission through the ear. Hear Res 272:30-41. CrossRef Medline

Mardia KV (1972) Statistics of directional data. Academic, New York.

McAlpine D (2005) Creating sense of auditory space. J Physiol 566:21-28. CrossRef Medline

McAlpine D, Jiang D, Palmer AR (1996) Interaural delay sensitivity and the classification of low best-frequency binaural responses in the inferior colliculus of the guinea pig. Hear Res 97:136-152. CrossRef Medline

McAlpine D, Jiang D, Palmer AR (2001) A neural code for low-frequency sound localization in mammals. Nat Neurosci 4:396-401. CrossRef Medline

McLaughlin M, Verschooten E, Joris PX (2010) Oscillatory dipoles as a source of phase shifts in field potentials in the mammalian auditory brainstem. J Neurosci 30:13472-13487. CrossRef Medline

Patterson RD (1976) Auditory filter shapes derived with noise stimuli. J Acoust Soc Am 59:640-654. CrossRef Medline

Pecka M, Siveke I, Grothe B, Lesica NA (2010) Enhancement of ITD coding within the initial stages of the auditory pathway. J Neurophysiol 103:3846. CrossRef Medline

Pecka M, Brand A, Grothe B (2008) Interaural time difference processing in the mammalian medial superior olive: the role of glycinergic inhibition J Neurosci 28:6914-6925. CrossRef

Reale RA, Brugge JF (1990) Auditory cortical neurons are sensitive to static 
and continuously changing interaural phase cues. J Neurophysiol 64: 1247-1260. Medline

Recio-Spinoso A (2012) Enhancement and distortion in the temporal representation of sounds in the ventral cochlear nucleus of chinchillas and cats. PLoS One 7:e44286. CrossRef Medline

Rosowski JJ, Ravicz ME, Songer JE (2006) Structures that contribute to middle-ear admittance in chinchilla. J Comp Physiol A Neuroethol Sens Neural Behav Physiol 192:1287-1311. CrossRef Medline

Ruggero MA, Temchin AN (2005) Unexceptional sharpness of frequency tuning in the human cochlea. Proc Natl Acad Sci U S A 102:18614-18619. CrossRef Medline

Rupert A, Moushegian G, Whitcomb MA (1966) Superior-olivary response patterns to monaural and binaural clicks. J Acoust Soc Am 39:1069-1076. CrossRef Medline

Schroeder MR (1977) New viewpoints in binaural interactions. In: Psychophysics and physiology of hearing (Evans EF, Wilson JP, eds), pp 455467. New York: Academic.

Scott LL, Mathews PJ, Golding NL (2010) Perisomatic voltage-gated sodium channels actively maintain linear synaptic integration in principal neurons of the medial superior olive. J Neurosci 30:2039-2050. CrossRef Medline

Shamma SA, Shen NM, Gopalaswamy P (1989) Stereausis: binaural processing without neural delays. J Acoust Soc Am 86:989-1006. CrossRef Medline

Siveke I, Pecka M, Seidl AH, Baudoux S, Grothe B (2006) Binaural response properties of low-frequency neurons in the gerbil dorsal nucleus of the lateral lemniscus. J Neurophysiol 96:1425-1440. CrossRef Medline

Smith PH, Joris PX, Yin TC (1993) Projections of physiologically characterized spherical bushy cell axons from the cochlear nucleus of the cat: evidence for delay lines to the medial superior olive. J Comp Neurol 331: 245-260. CrossRef Medline

Smith ZM, Delgutte B (2007) Sensitivity to interaural time differences in the inferior colliculus with bilateral cochlear implants. J Neurosci 27:67406750. CrossRef Medline

Spitzer MW, Semple MN (1995) Neurons sensitive to interaural phase disparity in gerbil superior olive: diverse monaural and temporal response properties. J Neurophysiol 73:1668-1690. Medline

Temchin AN, Ruggero MA (2010) Phase-locked responses to tones of chinchilla auditory nerve fibers: implications for apical cochlear mechanics. J Assoc Res Otolaryngol 11:297-318. CrossRef Medline

Tollin DJ, Yin TC (2005) Interaural phase and level difference sensitivity in low-frequency neurons in the lateral superior olive. J Neurosci 25:10648 10657. CrossRef Medline

van Bergeijk WA (1962) Variation on a theme of von Bekesy: a model of binaural interaction. J Acoust Soc Am 34:1431-1437. CrossRef

van der Heijden M, Lorteije JA, Plauška A, Roberts MT, Golding NL, Borst JG (2013) Directional hearing by linear summation of binaural inputs at the medial superior olive. Neuron 78:936-948. CrossRef Medline

Von Békésy G (1960) Experiments in hearing. New York: McGraw-Hill.

von Bismark G, Pfeiffer RR (1967) On the sound pressure transformation from free-field to eardrum. J Acoust Soc Am 42:1156. CrossRef

Webster DB, Plassmann W (1992) Parallel evolution of low-frequency sensitivity in old world and new world desert rodents. In: The evolutionary biology of hearing (Webster DB, Fay RR, Popper AN, eds), pp 633-636. New York: Springer.

Winer JA, Schreiner CE (2005) The inferior colliculus. New York: Springer.

Yin TC, Chan JC (1990) Interaural time sensitivity in medial superior olive of cat. J Neurophysiol 64:465-488. Medline

Yin TC, Kuwada S (1983) Binaural interaction in low-frequency neurons in inferior colliculus of the cat III. Effects of changing frequency. J Neurophysiol 50:1020-1042. Medline

Yin TC, Chan JC, Irvine DR (1986) Effects of interaural time delays of noise stimuli on low-frequency cells in the cat's inferior colliculus. I. Responses to wideband noise. J Neurophysiol 55:280-300. Medline 